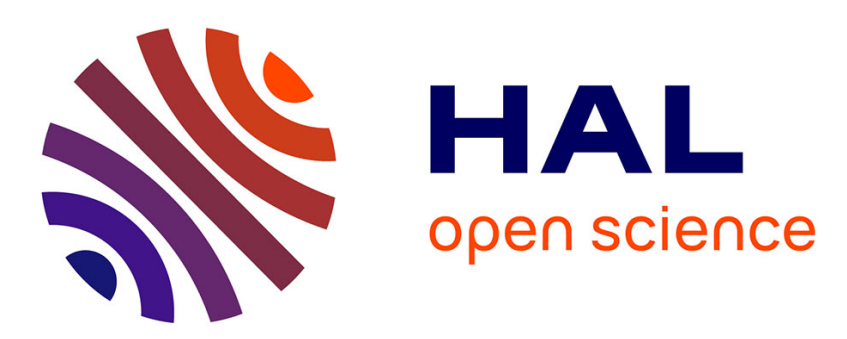

\title{
On UW-based Transmission for MIMO Multi-Carriers with Spatial Multiplexing
}

Shahab Ehsanfar, Marwa Chafii, Gerhard Fettweis

\section{To cite this version:}

Shahab Ehsanfar, Marwa Chafii, Gerhard Fettweis. On UW-based Transmission for MIMO MultiCarriers with Spatial Multiplexing. IEEE Transactions on Wireless Communications, In press. hal02617383

\section{HAL Id: hal-02617383 \\ https://hal.science/hal-02617383}

Submitted on 25 May 2020

HAL is a multi-disciplinary open access archive for the deposit and dissemination of scientific research documents, whether they are published or not. The documents may come from teaching and research institutions in France or abroad, or from public or private research centers.
L'archive ouverte pluridisciplinaire HAL, est destinée au dépôt et à la diffusion de documents scientifiques de niveau recherche, publiés ou non, émanant des établissements d'enseignement et de recherche français ou étrangers, des laboratoires publics ou privés. 


\title{
On UW-based Transmission for MIMO Multi-Carriers with Spatial Multiplexing
}

\author{
Shahab Ehsanfar, Marwa Chafii, IEEE Member, Gerhard Fettweis, IEEE Fellow
}

\begin{abstract}
In this paper, we design a frame structure for unique word (UW) based transmission of multiple-input-multiple-output (MIMO) systems under doubly-dispersive wireless channel conditions. We elaborate an energy and spectral efficiency analysis of a MIMO UW-based system vs. a conventional MIMO cyclic prefix (CP)-based system. Considering the UW-based transmission for a MIMO multi-carrier, we derive its signal processing algorithms for channel estimation and joint channelequalization-and-demodulation. Through theoretical derivations as well as extensive simulations, we show that the proposed MIMO UW-based system significantly outperforms the state-ofthe-art approaches.
\end{abstract}

\section{INTRODUCTION}

$\mathbf{T}$ OWARDS the sixth generation (6G) cellular networks, new applications and use-cases are being enabled for the vertical markets, while also new challenges for a holistic approach and more efficient service provisioning are being defined. Thanks to the flexible physical layer definitions in latest release of fifth generation (5G) New Radio (NR) [1], diverse services namely enhanced-mobile-broadband, ultrareliable-low-latency-communication, and massive machinetype-communication are envisioned to coexist within the same time interval and the same frequency band. A major advancement for realizing this coexistence is the massive multipleinput multiple-output (MIMO) technology which can be used for three key concepts, i.e., beamforming, spatial diversity and spatial multiplexing. While employing the MIMO technology for spatial diversity or spatial multiplexing, the mobility of the users becomes limited because a large number of channel parameters requires a long training sequence that together with the data packet, must fit within the channel coherence time.

Unique-word (UW) transmission is a promising candidate that facilitates per-block synchronization and channel estimation [2], [3], [4]. The main concept is to replace the cyclic prefix $(\mathrm{CP})$ of random nature by a deterministic sequence that is known to both transmitter and receiver. Hence, maintaining the advantages of $\mathrm{CP}$ in terms of robustness against multipath channels, UW allows fast channel tracking in highly mobile

Manuscript received December 22, 2019; revised March 24, 2020; accepted May 17, 2020. The computations were performed at the Center for Information Services and High Performance Computing (ZIH) of TU Dresden. This work has received funding from the European Union's Horizon 2020 research and innovation program under grant agreements no. 777137 (5GRANGE project) and no. 732174 (Orca project). Authors also acknowledge the Paris Seine Initiative for the support of the project through the ASIA Chair of Excellence Grant (PIA/ANR-16-IDEX-0008).

Authors are with the Vodafone Chair Mobile Communication Systems, Technische Universität (TU) Dresden, 01187 Dresden, Germany. Marwa Chafii is also with ETIS UMR 8051, CY Cergy Paris Université, ENSEA, CNRS, France. (e-mail: shahab.ehsanfar@ifn.et.tu-dresden.de; marwa.chafii@ensea.fr; fettweis@ifn.et.tu-dresden.de) scenarios where the underlying wireless channel is extremely doubly-dispersive. A number of studies have been proposed for channel estimation and equalization of UW-based single and multi-carrier systems. For instance, [4] and [5] proposed different approaches of channel estimation in single-input single-output (SISO) UW-single-carrier (SC) systems. The performance analysis, however, was performed under lowmobility channel conditions. In [6] and [7], an orthogonal frequency division multiplexing (OFDM)-based approach of UW generation [8] was extended to discrete Fourier transform (DFT) spread OFDM (DFT-s-OFDM) and its corresponding out-of-band (OOB) emission and peak-to-average power ratio (PAPR) were analyzed. Similarly in [9], a UW-based generalized frequency division multiplexing (GFDM) was proposed in order to maintain GFDM's low OOB emission and the performance was evaluated in block fading conditions. In [10], embedding a Zadoff-Chu sequence within the GFDM signal, a UW-based frame structure has been designed for vehicular communications. Nevertheless, all the above related works were focusing on SISO systems and their approaches are not straightforwardly applicable to MIMO multi-carriers.

In MIMO systems with large number of antennas, the receiver design becomes more challenging because significant number of factors must be taken into account. Indeed, by linearly increasing the number of transmit (Tx) antennas, the number of channel parameters increases quadratically [11]. Thus, in order to avoid significant increase of overhead signals and maintain a reasonable efficiency, a careful system design is indispensable. In addition, unlike SISO systems that have a wide range of deterministic sequences to be used as UW, in MIMO, the choices of the UW sequences are narrowed down to a smaller range. In fact, optimum UW sequences transmitted from different $\mathrm{Tx}$ antennas must jointly obey certain criteria in terms of synchronization, channel estimation, PAPR and OOB emission. Although, [12] and [13] proposed MIMO detection techniques for UW-OFDM systems, genieaided channel knowledge was being assumed and thus, the overhead of the system with respect to MIMO CP-OFDM pilots was not fully analyzed, and also the impact of the choice of UW sequences on signal PAPR and OOB radiation was ignored. To the best of our knowledge, no MIMO UWbased system design exists in literature, that can flexibly adopt any waveform (whether single or multi-carrier system) and can jointly consider MIMO UW-based channel estimation and equalization.

In this paper, we provide the following contributions to enable a robust UW-aided data transmission of MIMO transceivers in broadband mobile channels:

- By taking into account the UW frame design of [2] as a 
benchmark, we extend the frame structure to MIMO technology where the number of channel parameters are also being taken into account. Considering a doubly-dispersive channel condition, we analyze the overhead requirements of a UW-based system from synchronization and channel estimation perspectives-i.e. partially published in [14] and we compare its energy and spectral efficiency with respect to an equivalent pilot-aided CP-OFDM system design.

- Considering a non-orthogonal multi-carrier that not only focuses on robust data transmission, but also controls the transmit signal impairments, we analyze the MIMO UW sequences in terms of OOB radiation for GFDM.

- As the Payload-UW blocks are being transmitted through a highly frequency selective and time-variant channel, we derive the Wiener-Hopf filters for the corresponding MIMO linear minimum mean square error (LMMSE) channel estimation (CE). Proposing a frame structure that considers circularly rotating the UW slots of the MIMO antennas, we also analyze its CE performance with respect to the state-of-the-art (SoA) solutions.

- Proposing an equalization (EQ.) technique that reconstructs a cyclic signal at the receiver side, we derive its component-wise conditionally unbiased (CWCU) LMMSE equalizer with imperfect channel knowledge. Due to the cyclic signal reconstruction at the receiver side, our equalization technique allows the payload and UW sequences to be designed completely independent at the transmitter side. Therefore, the approach can embed the UW sequences to any single and multi-carrier system (e.g single carrier frequency domain equalization (SC-FDE), OFDM, GFDM), and maintain all their matrix structure advantages for low complexity detection approaches, as they were to be used in a CP-based system.

The remainder of this paper is organized as follows: In Sec. II, we review the SoA UW-based transmissions in SISO systems and we also describe the MIMO transmission scenario. In Sec. III, considering a MIMO UW-based frame design and a pilot-aided CP-OFDM system, we analyze the efficiency of the two systems. In Sec. IV, we investigate the OOB emission of the proposed UW sequences within the context of GFDM system. The channel estimation and joint channel-equalization-and-demodulation of the proposed UWbased system are derived in sections V and VI, respectively. The simulations and numerical results are provided in Sec. VII. Finally, the conclusions are drawn in Sec. VIII.

\section{Notations}

Column-vectors are denoted by vector sign $\vec{X}$ and matrices by boldface $\mathbf{X}$. Time domain signals are represented by lowercase letters while normal font uppercase letters are used for DFT domain signals. $\mathbb{E}[\cdot]$ is the expectation operator. $X$ modulo $Y$ is denoted by $X \bmod Y$. The conjugate, transpose and Hermitian transpose of $\boldsymbol{X}$ are denoted by $\boldsymbol{X}^{*}, \boldsymbol{X}^{T}$ and $\boldsymbol{X}^{H}$, respectively. $\boldsymbol{X} \otimes \boldsymbol{Y}$ and $\boldsymbol{X} \circ \boldsymbol{Y}$ are the Kronecker and Hadamard products [15] of matrices $\boldsymbol{X}$ and $\boldsymbol{Y}$, respectively. $\overrightarrow{\vec{Y}}$ is the element-wise division of vector $\vec{X}$ over $\vec{Y} \cdot \operatorname{diag}(\vec{X})$ is a diagonal matrix whose diagonal entries are the entries of the column vector $\vec{X}$. vec $(\boldsymbol{X})$ is the operation of stacking the columns of $\boldsymbol{X}$ on top of one another. The matrices $\boldsymbol{F}_{n}$ and $\boldsymbol{I}_{n}$ are the unitary DFT and the identity matrices of size $n \times n$, respectively. The matrix $\mathbf{0}_{m \times n}$ is an all zero matrix of size $m \times n . \overrightarrow{0}_{n}$ and $\overrightarrow{1}_{n}$ are column vectors of size $n$ with all zero and one entries, respectively. The distribution of a complex multivariate Gaussian random vector $\vec{X}$ with mean $\vec{\mu}=\mathbb{E}[\vec{X}]$ and covariance matrix $\boldsymbol{\Sigma}=\mathbb{E}\left[(\vec{X}-\vec{\mu})(\vec{X}-\mu)^{H}\right]$ is denoted by $\mathcal{N}_{C}(\vec{\mu}, \boldsymbol{\Sigma})$.

\section{State of The ART AND System Basics}

\section{A. Unique Words in SISO Systems}

In a UW-based system [2], instead of a $\mathrm{CP}$, a deterministic sequence is added as a prefix and also as a suffix around the transmission block. In this case, in order to keep the fast Fourier transform (FFT) size on the cyclic part of the receive (Rx) signal, the symbol duration reduces from $N+N_{\text {cp }}$ to $N$ samples [2]. Here, $N_{\mathrm{cp}}$ is the number of time-domain samples of the CP, and $N$ denotes the data FFT-size. Similar to CPbased systems, if the UW length $N_{u}$ is longer than the channel delay spread with length $L$, and also the channel remains constant during the block duration of $N$ samples, the receiver can interpret a circular channel transfer function and perform FFT-based channel estimation and equalization approaches. Moreover, since UW sequences are deterministic and known at the receiver, per-block synchronization can be achieved.

From a channel estimation perspective, if the UW length $N_{\mathrm{u}}$ is set to $L$ samples of the channel delay spread length, the UW observations would always be exposed to interference, because taking the FFT of the frame over $N=N_{d}+N_{\mathrm{u}}$ samples of $T_{\mathrm{FFT}}$ duration, payload becomes overlapped with UW sequences in frequency domain. In [4], authors stated that for a deterministic channel estimation method, a necessary and sufficient condition for channel identifiability is having the UW length to be at least twice of the channel length (i.e. [4] $N_{\mathrm{u}} \geq 2 L$ ) while also the first and the second half of the time domain UW signal must be identical, such that the channel on the second half looks circulant. Although, in order to relax the constraint on UW length to $N_{\mathrm{u}} \geq L$, they suggested to equalize the data and remove the associated interference from the UW sequence. The performance of the approach in [4] was not compared to an equivalent CP-based system and moreover, it is also only applicable to low-mobility channel conditions.

The generation of the unique word has been discussed differently in individual transmission schemes. For instance, in a UW-SC system, a Zadoff-Chu sequence can be attached to the $N-N_{u}$ length time domain SC signal [4]. In UWOFDM [8] and UW-DFT-s-OFDM [7] systems, a set of redundant subcarriers which cannot be used for data transmission have been considered in order to generate the UW sequence. In addition, since the output of the modulation matrix $\mathcal{M}$ in the linear transmit signal $\left[\begin{array}{ll}\vec{x}_{d}^{T} & \vec{x}_{\text {tail }}^{T}\end{array}\right]^{T}=\mathcal{M} \vec{d}$ might include the energy leakage of the random data to the tail part $\vec{x}_{\text {tail }}$, an approach has been proposed to suppress the energy leakage at the cost of higher transmitter complexity. The approach that we will consider in this work, is similar to the unique word 


\begin{tabular}{l|c|c|c|c|c|c|c|c|} 
Tx. 1: & $U_{0}$ & $U_{0}$ & Payload & $U_{0}$ & Payload & $U_{0}$ & Payload & $U_{0}$ \\
\cline { 2 - 9 } Tx. 2: & $U_{1}$ & $U_{1}$ & Payload & $U_{1}$ & Payload & $U_{1}$ & Payload & $U_{1}$ \\
Tx. 3: & $U_{2}$ & $U_{2}$ & Payload & $U_{2}$ & Payload & $U_{2}$ & Payload & $U_{2}$ \\
Tx. 4: & $U_{3}$ & $U_{3}$ & Payload & $U_{3}$ & Payload & $U_{3}$ & Payload \\
\cline { 2 - 7 }
\end{tabular}

(a) Basic UW

\begin{tabular}{l|c|c|c|c|c|c|c|c|} 
Tx. 1: & $U_{0}$ & $U_{0}$ & Payload & $U_{2}$ & Payload & $U_{0}$ & Payload \\
Tx. 2: & $U_{1}$ & $U_{1}$ & Payload & $U_{3}$ & Payload & $U_{1}$ & Payload \\
\hline \hline Tx. 3: & $U_{2}$ & $U_{2}$ & Payload & $U_{0}$ & Payload & $U_{2}$ & Payload \\
Tx. 4: & $U_{3}$ & $U_{3}$ & Payload & $U_{1}$ & Payload & $U_{3}$ & Payload & $U_{1}$ \\
\cline { 2 - 8 }
\end{tabular}

(b) Circ.-UW

Fig. 1. Example of the MIMO UW-based frames for $\mathfrak{I}=4$ Tx antennas.

generation in UW-SC systems (i.e. a time domain deterministic signal is attached to the time domain payload signal both as a prefix and suffix), although, by adopting a cyclic signal reconstruction analogously as in [16] at the receiver side, the proposed UW-based transmission scheme is applicable to any linear transmitter including OFDM, DFT-s-OFDM, GFDM, etc.

\section{B. Transmission over a Centralized MIMO Wireless Channel}

We consider that in a MIMO system, the Payload-UW blocks are structured according to Fig. 1, and they are simultaneously transmitted from $\mathfrak{I} \mathrm{Tx}$ antennas. In case of basic UW frames, the sequence $U_{i}^{\prime}$ that is transmitted after the payload block from antenna $i$ always remains identical with the sequence $U_{i}$ before the payload block, i.e., $U_{i \text {,basic }}^{\prime}=U_{i}$ (see Fig. 1a). On the other hand, as will be seen in (18), the covariance of the channel estimation directly depends on the observation sequence. Intuitively, one should expect that longer independent observations-i.e. more independent measurements-yield more accurate estimation. In other words, assuming that the channel remains static over the $U W$-Payload- $U W$ block, if one considers a joint LMMSE channel estimation over the two UW measurements, a plain figure of merit for the observation matrix is its condition number. Denoting $\boldsymbol{X}_{p}$ as the observation matrix associated to the UW sequences before the payload block, in case of basic UW, the condition number of the observation matrix associated to two UW measurements (before and after the payload block) $\boldsymbol{X}_{\mathrm{obs}}^{\prime}=\left[\boldsymbol{X}_{p}^{T}, \boldsymbol{X}_{p}^{T}\right]^{T}$ is equal to the condition number of $\boldsymbol{X}_{p}$. On the contrary, if the observation matrix $\boldsymbol{X}_{\mathrm{obs}}=\left[\boldsymbol{X}_{p}^{T}, \boldsymbol{X}_{p}^{\prime T}\right]^{T}$ achieves a condition number smaller than $\boldsymbol{X}_{\mathrm{obs}}^{\prime}$, further improvement in CE shall be expected. Nevertheless, the joint LMMSE of the channel over two delayed versions of the UW measurements, necessitates that the channel does not vary too quickly within the $U W$-Payload$U W$ block. In order to achieve such a condition within the UW-based frame design, we therefore consider an alternative case, in which the UW after the payload block circularly rotates by $\mathfrak{I} / 2$ (see Fig. 1b) for each antenna index $i$, i.e.

$$
U_{i, \text { circ }}^{\prime}=\left(\left(i+\frac{\mathfrak{I}}{2}\right) \bmod \mathfrak{I}\right) .
$$

Here, if $U_{i}^{\prime} \neq U_{i}$ and they have the least amount of correlation, one can achieve independent measurements over $U_{i}$ and $U_{i}^{\prime}$ and calculate the joint LMMSE of the two observed sequences for channel estimation. We also note that calculation of the joint LMMSE of the two UW sequences surrounding the payload block is also possible for the basic UW scheme, however, since the two measurements would be strongly correlated, the benefit of joint LMMSE of basic UW is expected to be less than that of Circ.-UW frames.

The transmission is initiated by sending a double UW sequence in form of a preamble, which assures a primary timefrequency synchronization and channel estimation. Afterwards blocks of Payload-UW with length $N$ are transmitted at a block-rate $F_{b}$. The transmit signals from $\mathfrak{I}$ Tx antennas pass through $\mathfrak{I} \times \mathfrak{Q}$ multi-path channels. Under the assumption of perfect synchronization, the receive signal $y_{q}[n]$ at antenna $q$ is characterized by the following expression:

$$
y_{q}[n]=\sum_{i=0}^{\mathfrak{I}-1} \sum_{\ell=0}^{L-1} h_{\ell, q, i}[n] x_{i}[n-\ell]+w_{q}[n],
$$

where $h_{\ell, q, i}[n]$ denotes the $\ell$-th tap of the channel impulse response's complex envelop at time sample $n$ between antennas $i$ and $q$. Further, $h_{\ell, q, i}[n]$ varies from sample-tosample and it is temporally correlated to $h_{\ell, q, i}\left[n^{\prime}\right]$ according to $R_{n, n^{\prime}}=\mathbb{E}\left(h_{\ell, q, i}\left[n^{\prime}\right]^{*} h_{\ell, q, i}[n]\right)$. The zero-mean additive white Gaussian noise (AWGN) process with variance $\sigma_{w}^{2}$ at Rx antenna $q$ is denoted by $w_{q}[n]$.

Collecting the receive samples $y_{q}[n]$ of an $N$-length Payload- $U W$ block in vector form $\vec{y}_{q}$ where $N=N_{d}+N_{\mathrm{u}}$, and assuming that the channel impulse response (CIR) has been estimated as $\hat{\vec{h}}_{q, i}$, the conventional equalization technique, e.g. [9] uses the circularity of the channel over the $N$-length Payload- $U W$ block. Thus, via an LMMSE based equalization technique, the estimated Tx signal is given by

$$
\begin{aligned}
\hat{\vec{x}}_{d} & =\overline{\boldsymbol{F}}_{N_{d}, N} \hat{\boldsymbol{H}}_{N}^{H}\left(\hat{\boldsymbol{H}}_{N} \hat{\boldsymbol{H}}_{N}^{H}+\sigma_{w}^{2}\right)^{-1}\left[\boldsymbol{F}_{N} \vec{y}_{q}\right]_{q=0: \mathfrak{Q}} . \\
\boldsymbol{\Sigma}_{x_{d}, x_{d}} & =\overline{\boldsymbol{F}}_{N_{d}, N} \hat{\boldsymbol{H}}_{N}^{H}\left(\hat{\boldsymbol{H}}_{N} \hat{\boldsymbol{H}}_{N}^{H}+\sigma_{w}^{2}\right)^{-1} \hat{\boldsymbol{H}}_{N}
\end{aligned}
$$




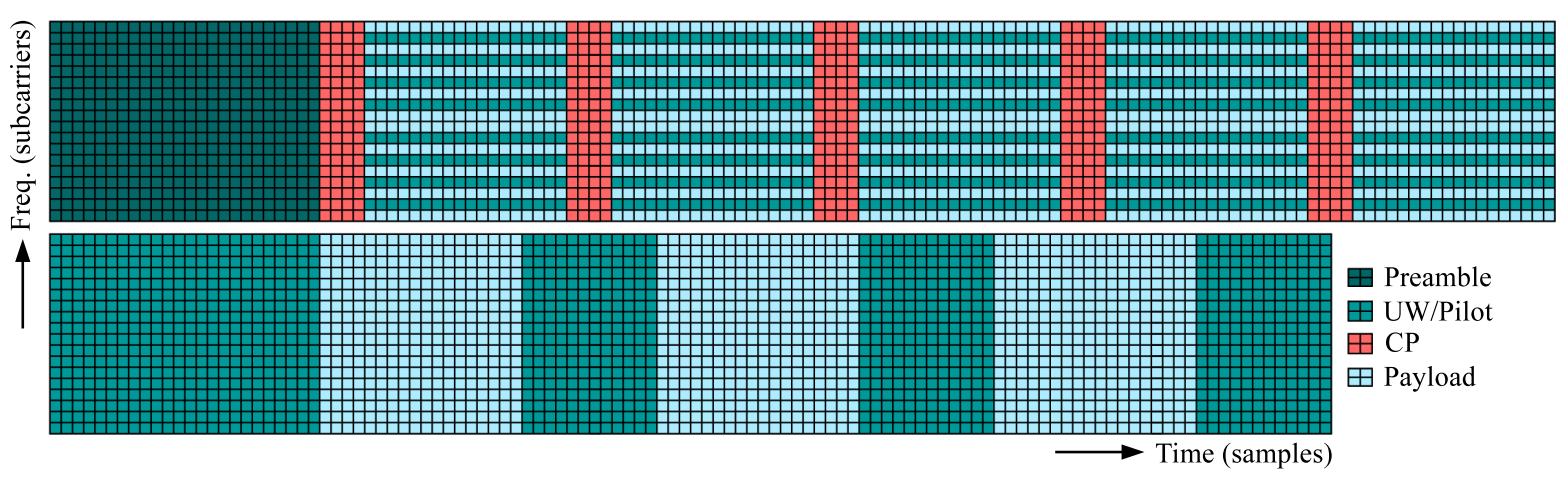

Fig. 2. Example of the frame structure in time-frequency resource grid. Here, each resource element is associated to the bandwidth of one subcarrier and the duration of one time sample. Without loss of generality, the impact of guard band insertion has been neglected in this figure. As one may see, the UW-based frame design, saves the time-frequency resources of one OFDM symbol for transmission of 100 complex data over a $2 \times 2$ MIMO channel.

where $\overline{\boldsymbol{F}}_{N_{d}, N}=\left(\boldsymbol{I}_{\mathfrak{I}} \otimes \boldsymbol{F}_{N_{d}, N}\right)$ and $\boldsymbol{F}_{N_{d}, N}$ includes only the first $N_{d}$ rows of $\boldsymbol{F}_{N}$. The frequency domain estimated channel matrix $\hat{\boldsymbol{H}}_{N} \in \mathbb{C}^{\mathfrak{Q} N \times \mathfrak{Q} N}$ denotes the MIMO version of $\mathfrak{Q} \times$ $\mathfrak{I}$ matrices $\hat{\boldsymbol{H}}_{q, i, N}=\operatorname{diag}\left(\sqrt{N} \boldsymbol{F}_{N, L} \hat{\vec{h}}_{q, i}\right)$. Afterwards, a separate demodulation technique similar to [9] might calculate the decision metrics for the input of the decoder.

\section{FRAME DESIGN AND EFFICIENCY ANALYSIS}

\section{A. Illustrative Scenario}

For clarity, consider an extreme scenario of a spatial multiplexing $2 \times 2$ MIMO system, in which, 100 complex data ${ }^{1}$ (i.e. 50 per Tx. antenna) must be transmitted by $K=18$ subcarriers over a frequency selective-with $L=4$ taps-and fast fading channel. In [17], it is recommended that for a CPOFDM system in a time-variant channel, it is necessary to employ space-frequency pilots pattern, i.e. $\Im L$ pilot insertion into every OFDM symbol. In this case $\mathfrak{I} L=8$ channel taps must be estimated and therefore, 8 pilot subcarriers must be inserted into every OFDM symbol. From a total number of 18 subcarriers, each OFDM symbol can carry 10 data subcarriers. Hence, because of the two streams being simultaneously transmitted over a $2 \times 2$ MIMO channel, five OFDM symbols would be needed to carry $10 \times 2 \times 5=100$ complex symbols over $5(18+4)=110$ time resources. The frame design for such an OFDM system is depicted in the upper part of Fig. 2. Considering that the power is doubled over the two MIMO streams, the energy efficiency of this frame design is $\eta_{\mathcal{E} \text {, Pilots }}=\frac{100}{2 \times 110}=45 \%$.

Now, consider the same explanatory scenario, in which, the knowledge of UW sequences shall be exploited for channel estimation (and also synchronization), and therefore, no pilot insertion is necessary. In this case, we design the length of the UW sequence to be $N_{\mathrm{u}} \geq(\mathfrak{I}+1) L$, such that the first $L$ samples shall be ignored (due to the inter-block-interference (IBI) from the previous block), and the rest of $\left(N_{\mathrm{u}}-L\right) \geq \mathfrak{I} L$ samples would be used to estimate $\mathfrak{I} L$ channel parameters. Unlike the approach in [2], which considers the length of Payload- $U W$ as the FFT size and thus reduces the payload size

${ }^{1}$ Complex data is referred to any complex value from a modulation alphabet, e.g. $2^{\mu}$-QAM. For instance, one may assume 100 bits via $1 / 2$ code-rate QPSK.
$N_{d}$, we keep the FFT size for $N_{d}$ samples without including the UW length in it, and for its corresponding equalization, we will reconstruct a cyclic signal at the receiver side and elaborate further in Sec. VI. In addition, at the beginning of a transmission, we consider a double UW sequence for primary synchronization, which is equivalent to the preamble of the CP-OFDM system. For the rest of the blocks, we consider only a single UW sequence. Note that here, if we use double UW sequence for all blocks, the overhead becomes significant, and if we use double half-length UW sequences, the signalto-noise ratio (SNR) for synchronization becomes half. The frame design of such a UW-based system is depicted in lower part of Fig. 2. There, we can see that 12 time samples are considered for each UW sequence, while all the 18 subcarriers of the data symbol are actively used for data transmission. In this case, for three UW-based symbols, $18 \times 2 \times 3=108$ complex symbols are transmitted over $3(18+12)=90$ time resources. Therefore, the energy efficiency of the UW-based frame design becomes $\eta_{\mathcal{E}, \mathrm{UW}}=\frac{108}{2 \times 90}=60 \%$, and thus the time resource of one OFDM symbol is being saved. The detailed efficiency analysis of such frame design is further discussed in the following.

\section{B. CP vs. UW Efficiency analysis}

Consider that for primary synchronization, a preamble consisting of two repetitive deterministic sequences shall be used for both CP-OFDM and UW-OFDM. The CP-OFDM employs a preamble for $B_{\mathrm{CP}}$ number of OFDM blocks, while in UWOFDM the preamble is used for $B_{\mathrm{UW}} \geq B_{\mathrm{CP}}$. Thanks to the unique word sequences after each payload block in UWOFDM, per-block-synchronization allows $B_{\mathrm{UW}}$ to be much larger than $B_{\mathrm{CP}}$.

Summing up the overhead requirements that were discussed in Sec. III-A and beginning of this section, the numbers of overhead samples for pilot-aided CP-OFDM and UW-OFDM follow ${ }^{2}$ :

$$
\xi_{\mathrm{CP}+\text { Pilots }}=\underbrace{N_{p}}_{\text {Preamble }} N_{d}+B_{\mathrm{CP}}(\underbrace{L}_{\mathrm{CP}} N_{d}+\underbrace{\Im L}_{\text {Pilots }} N_{d}),(5)
$$

${ }^{2}$ Assuming full subcarrier allocation for $N_{d}$-point DFT size, $L$ time domain samples of CP in (5) occupy $N_{d}$ frequency bins, whereas, $\mathfrak{I} L$ frequency domain pilots are spread over $N_{d}$ time samples. 


$$
\xi_{\mathrm{UW}}=N_{p} N_{d}+B_{\mathrm{UW}} N_{d} N_{\mathrm{u}},
$$

respectively. Here, $N_{p}$ is the preamble size, and for a UWbased system, we set it to $N_{p}=2 N_{\mathrm{u}}$ for two repeated UW sequences. Assuming $N_{\mathrm{u}}=(\mathfrak{I}+1) L$ and comparing (5) and (6), one may notice that if the number of transmission blocks $B_{\mathrm{CP}}$ and $B_{\mathrm{UW}}$ are equal, the number of overhead samples for CP-OFDM and UW-OFDM becomes equal too, i.e. $\xi_{\mathrm{CP}+\mathrm{Pilots}}=$ $\xi_{\mathrm{UW}}$ for $B_{\mathrm{CP}}=B_{\mathrm{UW}}$.

On the other hand, the total number of resources for both systems follow:

$$
\begin{aligned}
S_{\mathrm{CP}} & =N_{p} N_{d}+B_{\mathrm{CP}} N_{d}\left(L+N_{d}\right), \\
S_{\mathrm{UW}} & =N_{p} N_{d}+B_{\mathrm{UW}} N_{d}\left(N_{\mathrm{u}}+N_{d}\right) .
\end{aligned}
$$

Clearly, the resource size $S_{\mathrm{UW}}$ for UW-OFDM is always larger than $S_{\mathrm{CP}}$ for CP-OFDM, even if the number of blocks $B_{\mathrm{UW}}$ and $B_{\mathrm{CP}}$ are equal. Consequently, we calculate the energy efficiency by taking the ratio of the number of resources dedicated to useful data transmission with respect to the total number of resources, i.e.,

$$
\begin{aligned}
\eta_{\mathcal{E}, \text { Pilots }} & =\frac{S_{\mathrm{CP}}-\xi_{\mathrm{CP}+\text { Pilots }}}{S_{\mathrm{CP}}}, \\
\eta_{\mathcal{E}, \mathrm{UW}} & =\frac{S_{\mathrm{UW}}-\xi_{\mathrm{UW}}}{S_{\mathrm{UW}}} .
\end{aligned}
$$

The above energy efficiency of the two systems does not take into account the gain with respect to the modulation order $\mu$ and spatial multiplexing with $\mathfrak{I} \mathrm{Tx}$ antennas. Therefore, assuming high SNR conditions, (9) and (10) shall be mapped into the upper bound of spectral efficiency (in bit/second/Hertz) via

$$
\begin{gathered}
\eta_{S, \text { Pilots }}=\frac{\Im \mu B_{\mathrm{CP}}\left(N_{d}-\Im L\right)}{T_{F, \mathrm{CP}} \mathcal{B}}, \\
\eta_{S, \mathrm{UW}}=\frac{\Im \mu B_{\mathrm{UW}} N_{d}}{T_{F, \mathrm{UW}} \mathcal{B}},
\end{gathered}
$$

where $T_{F, \mathfrak{M}}=S_{\mathfrak{M}} T_{s}$ denotes the frame duration for $\mathfrak{M} \in$ $\{\mathrm{CP}, \mathrm{UW}\}$. Furthermore, $\mathcal{B}$ denotes the bandwidth of the system and in case of full subcarrier allocation we have $\mathcal{B}=F_{s}$. Here, $F_{s}=\frac{1}{T_{s}}$ denotes the sampling frequency. Note that (11) and (12) describe only an upper bound to the spectral efficiency, because, due to further impairments, e.g. guard band insertion, channel codes and packet error rate (PER), the efficiency might degrade. Nevertheless, if the modulation order $\mu$ has been chosen appropriately by taking into account the coherence bandwidth and coherence time of the underlying scenario, $\eta_{S \text {,Pilots }}$ and $\eta_{S \text {,Uw }}$ provide fair figures of merit for efficiency comparison.

\section{SEQUenCES FOR UW AND OOB RADIATION}

In order to select an optimal choice of UW sequences for MIMO wireless transmission, the following aspects must be taken into account:

- An accurate MIMO synchronization shall be achieved if and only if the periodic auto-correlation functions of the sequences are Kronecker's Delta function $\delta[n]$, and also, their periodic cross-correlation functions-of the sequences being transmitted from different antennasare constant values which yield to minimized maximum absolute value [18],[19].

- From channel estimation perspective, the UW sequences must cover the entire band where the payload is being transmitted, and also they must have constant-modulus DFT (i.e. optimum in estimator's mean squared error (MSE) sense).

- In order to maintain low PAPR, the finite sequences must be of constant magnitude in their discrete time domain signal.

- Near optimum sequences from OOB emission perspective should not have abrupt changes in their real and imaginary components of the time domain signal.

Orthogonal Polyphase Sequences: Similar to [18], we use the polyphase sequences proposed in [19] to generate the unique words for different Tx antennas. Thus, the UW sequence $x_{\mathrm{u}, i}[n]$ from Tx antenna $i$ is given by

$$
x_{\mathrm{u}, i}[n]=\exp \left(\frac{j 2 \pi i n_{0} n_{1}}{\sqrt{N_{\mathrm{u}}}}\right),
$$

where, $n=n_{0} \sqrt{N_{\mathrm{u}}}+n_{1}$, for $n_{0} \in \mathcal{S}, n_{1} \in \mathcal{S}$, $\mathcal{S}=\left\{0, \cdots, \sqrt{N_{\mathrm{u}}}-1\right\}$. Collecting the samples of $x_{\mathrm{u}, i}[n]$ in form of a vector notation $\vec{x}_{\mathrm{u}, i}, N_{\mathrm{u}}$ is the length of $\vec{x}_{\mathrm{u}, i}$ and the square root $\sqrt{N_{\mathrm{u}}}$ must be a prime number [19]. We note that, the periodic auto-correlation function of $\vec{x}_{\mathrm{u}, i}$ is a Kronecker's Delta function $\delta[n]$, while the periodic crosscorrelation of $\vec{x}_{\mathrm{u}, i}$ and $\vec{x}_{\mathrm{u}, i^{\prime} \neq i}$ is a constant value equal to $1 / \sqrt{N_{\mathrm{u}}}$ [19]. Moreover, the magnitude of $\vec{x}_{\mathrm{u}, i}$ in both time and DFT domains is constant. In the following, we show that integrating the sequence with a low OOB emitting multicarrier can still preserve the desired signal characteristics.

\section{A. Waveform Engineering for UW Sequences combined with GFDM}

Let $\vec{x}_{i}=\left[\begin{array}{ll}\vec{x}_{d, i}^{T} & \vec{x}_{\mathrm{u}, i}^{T}\end{array}\right]^{T}$ be the transmit signal from Tx antenna $i$, where the payload vector $\vec{x}_{d, i}$ is linearly modulated by a multi-carrier system. In case of a flexible framework for waveform generation (i.e. GFDM), $\vec{x}_{d, i}$ is given by [20] $\vec{x}_{d, i}=\boldsymbol{A} \vec{d}_{i}$, where $\vec{d}_{i}=\operatorname{vec}\left(\mathbf{D}_{i}\right)$, in which $\mathbf{D}_{i} \in \mathbb{C}^{K \times M}$ is the allocation matrix for $K$ subcarriers and $M$ subsymbols, and its elements comprise data mapped to a modulation alphabet. Moreover, $\boldsymbol{A} \triangleq$ $\left(\vec{g}_{0,0}, \cdots, \vec{g}_{K-1,0}, \vec{g}_{0,1}, \vec{g}_{1,1}, \cdots, \vec{g}_{K-1, M-1}\right)$ is the modulation matrix of GFDM, where $\vec{g}_{k, m}=\left(g_{k, m}[n]\right)_{n=0,1}^{T}$ with $N_{d}=M K$, is the pros $\vec{g}_{k, m}=\left(g_{k, m}[n)_{n=0,1, \cdots, N_{d}-1}^{T}\right.$ $N_{d}=M K$, is the protype flter at subcarrier index $k$ and subsymbol index $m$. By properly choosing the prototype filter $\vec{g}_{0,0}$, various waveforms shall be generated, e.g. if $\vec{g}_{0,0}$ is a sinc function, the output of the multi-carrier modulation is DFT-s-OFDM, whereas the conventional circular pulse shaping approaches may employ different prototype filters as well.

A UW-GFDM block would maintain GFDM's low OOB emission, provided that the transition between the UW sequence and the GFDM signal is a smooth transition without any abrupt change of the signal. Such condition shall be 

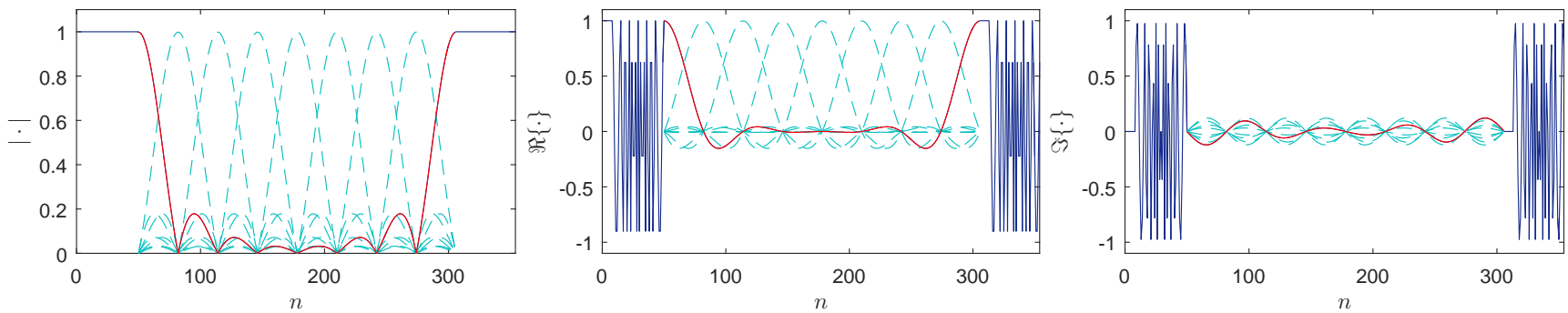

Fig. 3. UW-GFDM signal in time domain (dark blue: UW, red: first GFDM subsymbol, light blue: rest of GFDM subsymbols) at zeroth subcarrier. If (14) holds, the red curve remains constant and does not get influenced by random data.

achieved by setting the first GFDM subsymbol as a guard symbol (GS). Thus, we have

$$
d_{0, i}[k]=\delta\left[k_{p}\right]
$$

where $\left(\vec{d}_{0, i}\right)_{k=0: K-1}$ is the first column of $\mathbf{D}_{i}$ associated to the first subsymbol. The constant $k_{p}$ can be any subcarrier within the band. Here, the Kronecker's Delta function $\delta[\cdot]$, generates the shape of the time domain prototype filter as the time domain signal. If its energy is normalized to its maximum value, its time domain signal begins with $x_{d, i}[0]=1$ and also ends with $x_{d, i}\left[N_{d}-1\right]=1$. Having $x_{u, i}[0]=1$ and $x_{u, i}\left[N_{\mathrm{u}}-1\right] \approx 1$, a smooth transition between UW and GFDM signal is achieved. Fig. 3 shows an example of the signal in time domain, in which the GFDM signal is surrounded by two UW sequences. As one can see, the first GFDM subsymbol has the key impact on the transition between the UW and the GFDM block. Thus, having the first subsymbol as a GS, a smooth transition is achieved at the boundary of UW and GFDM blocks. Nevertheless, the abrupt changes of the real and imaginary components of the UW itself, would have negative impacts on the overall OOB radiation of the signal. Assuming the energy of $\delta\left[k_{p}\right]$ to be $\sqrt{K}$, (i.e. the normalization factor of the prototype filter $\vec{g}_{k, m}$ ), the GS insertion of the above approach yields an increase of overhead by the factor $\frac{1}{M \sqrt{K}}$.

\section{Channel Estimation}

Under the assumption of perfect time and frequency synchronization and also a block-fading situation, the received signal associated to the last IBI-free $\left(N_{\mathrm{u}}-L\right)$ samples of the UW $\vec{x}_{\mathrm{u}, i}$-defined in (13)—at antenna $q$ and block $b$ is given by

$\vec{y}_{p, q, b}=\left[\boldsymbol{h}_{\mathrm{Tpl}, q, 0, b}, \cdots, \boldsymbol{h}_{\mathrm{Tpl}, q, \mathfrak{I}-1, b}\right]\left[\vec{x}_{p, 0}^{T}, \cdots, \vec{x}_{p, \mathfrak{I}-1}^{T}\right]^{T}+\vec{w}_{q}$

where, $\vec{x}_{p, i}=\left(\vec{x}_{\mathrm{u}, i}\right)_{L: N_{\mathrm{u}}-1}$, and $\boldsymbol{h}_{\mathrm{Tpl}, q, i, b} \in \mathbb{C}^{\left(N_{\mathrm{u}}-L\right) \times\left(N_{\mathrm{u}}-L\right)}$ is a lower triangular Toeplitz channel matrix with $\vec{h}_{q, i, b}$ denoting the parameters vector of $L$-length CIR-on its first column. Rearranging the matrix-vector notations, we get

$$
\vec{y}_{p, q, b}=\boldsymbol{X}_{p} \vec{h}_{q, b}+\vec{w}_{q},
$$

where $\boldsymbol{X}_{p}=\left[\boldsymbol{X}_{p, 0}, \cdots, \boldsymbol{X}_{p, \mathfrak{I}-1}\right] \in \mathbb{C}^{\left(N_{u}-L\right) \times \mathfrak{I} L}$ is the observation matrix with $\boldsymbol{X}_{p, i} \in \mathbb{C}^{\left(N_{\mathrm{u}}-L\right) \times L}$ being the last $\left(N_{\mathrm{u}}-L\right)$ rows and first $L$ columns of $\boldsymbol{X}_{u, i}$. Here, $\boldsymbol{X}_{u, i} \in \mathbb{C}^{N_{\mathrm{u}} \times N_{\mathrm{u}}}$ is a lower triangular Toeplitz matrix with $\vec{x}_{\mathrm{u}, i}$ on its first column. Further, $\vec{h}_{q, b}=\left[\vec{h}_{q, i, b}^{T}\right]_{i=0: \mathfrak{I}-1}^{T} \in \mathbb{C}^{\mathfrak{I} L}$, and $\vec{w}_{q} \in \mathbb{C}^{N_{\mathrm{u}}-L}$ denotes the AWGN process of variance $\sigma_{w}^{2}$.

\section{A. MIMO UW-based LMMSE CE}

The LMMSE estimation of $\vec{h}_{q, b}$ and its covariance follow [21]

$$
\begin{aligned}
\hat{\vec{h}}_{q, b} & =\left(\sigma_{w}^{2} \boldsymbol{\Sigma}_{h h}^{-1}+\boldsymbol{X}_{\mathrm{obs}}^{H} \boldsymbol{X}_{\mathrm{obs}}\right)^{-1} \boldsymbol{X}_{\mathrm{obs}}^{H} \vec{y}_{\mathrm{obs}, q, b} \\
\widehat{\boldsymbol{\Sigma}}_{h h} & =\boldsymbol{\Sigma}_{h h}-\left(\sigma_{w}^{2} \boldsymbol{\Sigma}_{h h}^{-1}+\boldsymbol{X}_{\mathrm{obs}}^{H} \boldsymbol{X}_{\mathrm{obs}}\right)^{-1} \boldsymbol{X}_{\mathrm{obs}}^{H} \boldsymbol{X}_{\mathrm{obs}} \boldsymbol{\Sigma}_{h h}
\end{aligned}
$$

respectively. Here, $\boldsymbol{\Sigma}_{h h}=\operatorname{diag}\left(\overrightarrow{1}_{\mathfrak{I}} \otimes \overrightarrow{\mathcal{P}}\right)$ wherein, $\overrightarrow{\mathcal{P}} \in \mathbb{C}^{L}$ denotes the single power-delay-profile (PDP) between all Tx-Rx antenna pairs in a centralized MIMO system. We assume that $\overrightarrow{\mathcal{P}}$ and $\sigma_{w}^{2}$ are perfectly known at the receiver. If the receiver applies separate LMMSE CE to each UW measurement $\vec{y}_{\mathrm{obs}, q, b}^{\mathrm{basic}}=\vec{y}_{p, q, b}$, we have the observation matrix $\boldsymbol{X}_{\text {obs }}^{\text {basic }}=\boldsymbol{X}_{p}$, and once, an estimate $\hat{\vec{h}}_{q, b}=\left[\hat{\vec{h}}_{q, i, b}^{T}\right]_{i=1: \mathfrak{I}}^{T}$ for each of both UW sequences over the $U W$-Payload- $U W$ block is obtained, the two estimations are being averaged and fed to the equalization unit, i.e.

$$
\hat{\vec{h}}_{q, i, b, \mathrm{~B}}=0.5\left(\hat{\vec{h}}_{q, i, b-1}+\hat{\vec{h}}_{q, i, b}\right) .
$$

On the other hand, if Circ.-UW frames are being employed (as discussed in Sec. II-B and Fig. 1b), it is more beneficial that we apply joint LMMSE estimation on the two UW sequences in $U W$-Payload- $U W$ block. In this case, while the measurement $\vec{y}_{\text {obs }, q, b}^{\text {Circ-UW }}=\left[\vec{y}_{p, q, b-1}^{T}, \vec{y}_{p, q, b}^{T}\right]^{T}$ considers the concatenation of two IBI-free UW sequences (by ignoring the first $L$ samples), the observation matrix follows

$$
\boldsymbol{X}_{\mathrm{obs}}^{\text {circ.-UW }}=\left[\begin{array}{l}
\boldsymbol{X}_{p, 1}, \cdots, \boldsymbol{X}_{p, \frac{\mathfrak{I}}{2}}, \boldsymbol{X}_{p, \frac{\mathfrak{I}}{2}+1}, \cdots, \boldsymbol{X}_{p, \mathfrak{I}} \\
\boldsymbol{X}_{p, \frac{\mathfrak{I}}{2}+1}, \cdots, \boldsymbol{X}_{p, \mathfrak{I}}, \boldsymbol{X}_{p, 1}, \cdots, \boldsymbol{X}_{p, \frac{\mathfrak{I}}{2}}
\end{array}\right]
$$

An advantage of the above LMMSE estimation is that the observation signal $\vec{x}_{p, i}$ is interference-free from IBI perspective. However, it also has a drawback due to the partial selection of $\vec{x}_{\mathrm{u}, i}$. The sequence $\vec{x}_{\mathrm{u}, i}$ has a constant frequency magnitude which is an optimal sequence for channel estimation from MSE sense. By selecting the last $N_{\mathrm{u}}-L$ samples of $\vec{x}_{\mathrm{u}, i}$, the signal magnitude in frequency domain decreases at near edge subcarriers. Therefore, in case of a 


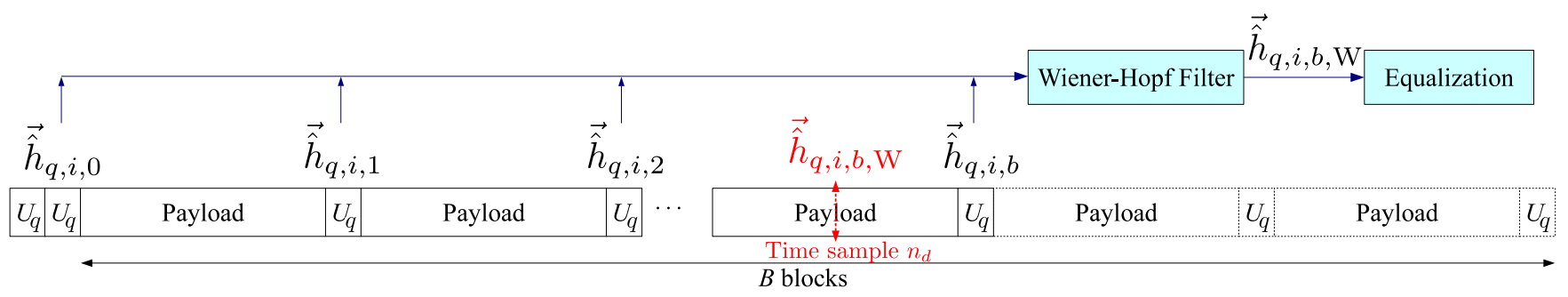

Fig. 4. Adaptive Filtering of the estimated CIRs for $b \leq B$ blocks

full subcarrier allocation, it would be recommended to use the whole $\vec{x}_{\mathrm{u}, i}$ sequence for channel estimation and apply iterative interference cancellation in analogous way as in [22], although, applying such an approach is beyond the scope of this paper.

\section{B. Adaptive Filtering}

Considering that $b \leq B$ number of Payload- $U W$ blocks have been transmitted after the preamble, and thus, $b+1$ channel estimations are available, we apply a Wiener-Hopf filter analogously as in [23] to improve the channel estimation at block $b$, although, with the main difference that the Wiener filter is being applied solely to use the estimated CIRs to obtain an improved estimation of the channel at the center ${ }^{3}$ of the payload block $b$.

Fig. 4 illustrates the filtering approach, in which, the $b+1$ channel estimations

$$
\hat{\boldsymbol{h}}_{q, i}=\left[\hat{\vec{h}}_{q, i, 0}, \hat{\vec{h}}_{q, i, 1}, \cdots, \hat{\vec{h}}_{q, i, b}\right]
$$

are used to calculate the CIR at time sample $n_{d}$ in the center of the $b$-th payload block. Once the estimations have been filtered, the resulting vector $\left(\hat{\vec{h}}_{q, i, b, \mathrm{~W}}\right)_{\ell=0: L-1}$ is being used for the equalization of the corresponding block. The adaptive filtering based on Wiener-Hopf Approach [21],[23] follows:

Let $\mathbf{R}_{h} \in \mathbb{C}^{(b+2) \times(b+2)}$ be the temporal auto-correlation matrix of the channel tap $\ell$ at the respective time samples, i.e.

$$
\begin{array}{r}
{\left[\mathbf{R}_{h}\right]_{n . n^{\prime}}=\mathbb{E}\left(h_{\ell, q, i}\left[n^{\prime}\right]^{*} h_{\ell, q, i}[n]\right)} \\
\quad \text { for } n, n^{\prime} \in\left\{n_{u, 0}, \cdots, n_{u, b-1}, n_{d}, n_{u, b}\right\},
\end{array}
$$

where the sample indexes $n_{d}$ and $n_{u, b}$ are the sample time indexes associated to the center of the $b$-th payload and UW, respectively. The filtered CIR's $\ell$-th tap at block $b$ is given by

$$
\hat{h}_{q, i, b, \mathrm{~W}}[\ell]=\left[\vec{R}_{h}\right]_{\mathcal{U}, b}^{H}\left(\mathbf{R}_{h}^{(\mathcal{U})}+\hat{\sigma}_{\ell}^{2} \boldsymbol{I}_{(b+1)}\right)^{-1} \overrightarrow{\hat{h}}_{q, i, \ell},
$$

where $\overrightarrow{\hat{h}}_{q, i, \ell}$ is the transposed $\ell$-th row of $\hat{\boldsymbol{h}}_{q, i}$, $\left[\vec{R}_{h}\right]_{\mathcal{U}, b} \subset \mathbf{R}_{h}^{(\mathcal{U}, d)}$ is the $b$-th column of $\mathbf{R}_{h}^{(\mathcal{U}, d)}$. The sub-matrix $\mathbf{R}_{h}^{(\mathcal{U}, d)} \subset \mathbf{R}_{h}$ contains the rows of $\mathbf{R}_{h}$ associated to the UW slots (i.e. without the row index associated to $n_{d}$ ). $\mathbf{R}_{h}^{(\mathcal{U})} \subset \mathbf{R}_{h}^{(\mathcal{U}, d)}$ contains the columns of $\mathbf{R}_{h}^{(\mathcal{U}, d)}$ associated to the UW slots. $\hat{\sigma}_{\ell}^{2}$ denotes the $\ell$-th diagonal element of $\widehat{\boldsymbol{\Sigma}}_{h h}$.

${ }^{3}$ With the assumption that the channel varies within the block duration.
Moreover, the covariance of the filtered CE associated to tap $\ell$ is given by

$$
\begin{aligned}
& \left(\widehat{\boldsymbol{\Sigma}}_{h h, \ell, \mathrm{W}}\right)_{q, i}= \\
& \left(\mathbf{R}_{h}-\mathbf{R}_{h}^{(\mathcal{U}, d)}{ }^{H}\left(\mathbf{R}_{h}^{(\mathcal{U})}+\hat{\sigma}_{\ell}^{2} \boldsymbol{I}_{(b+1)}\right)^{-1} \mathbf{R}_{h}^{(\mathcal{U}, d)}\right)_{q, i}
\end{aligned}
$$

Therefore, at block $b$, the CE covariance becomes

$$
\left(\widehat{\boldsymbol{\Sigma}}_{h h, \mathrm{~W}}[b]\right)_{q, i}=\left(\operatorname{diag}\left(\left[\left(\widehat{\boldsymbol{\Sigma}}_{h h, \ell, \mathrm{W}}[b, b]\right)_{\ell=0: L-1}\right]^{T}\right)\right)_{q, i} .
$$

Since the above Wiener-Hopf filter adopts the past and present measurements, it shall be viewed as application of a causal filter to the channel estimates. We also note that (23) is an application of LMMSE estimators and therefore it obeys the Bayesian approach by minimizing the global MSE. If the channel remains static, i.e. $\mathbb{E}\left(h_{\ell, q, i}\left[n^{\prime}\right]^{*} h_{\ell, q, i}[n]\right)=1$, the matrix $\left[\mathbf{R}_{h}\right]_{n . n^{\prime}}$ becomes an all ones matrix and thus, it averages all the elements in $\overrightarrow{\hat{h}}_{q, i, \ell}$ by also considering the weighting coefficient $\hat{\sigma}_{\ell}^{2}$ which defines the channel estimation MSE at tap $\ell$. On the contrary, if the channel is infinitely timevariant, i.e. $\mathbb{E}\left(h_{\ell, q, i}\left[n^{\prime}\right]^{*} h_{\ell, q, i}[n]\right) \approx 0$ for $n^{\prime} \neq n$, we would have $\left[\mathbf{R}_{h}\right]_{n . n^{\prime}} \approx \boldsymbol{I}_{b+2}$, and therefore, each element of $\overrightarrow{\hat{h}}_{q, i, \ell}$ is considered to be completely uncorrelated, and therefore, every estimation $\hat{\vec{h}}_{q, b}$ is treated to be independent. In case of extremely time-varying channels, the coefficient $\hat{\sigma}_{\ell}^{2}$ applies further weights to the auto-correlation matrix $\mathbf{R}_{h}^{(\mathcal{U})}$, which is beneficial if $\mathbf{R}_{h}^{(\mathcal{U})}$ is a singular matrix.

\section{EQUALIZATION WITH IMPERFECT CHANNEL KNOWLEDGE}

The state-of-the-art of EQ. techniques for UW-based multicarrier systems consider using the circularity of the channel over the Payload-UW block and equalize the payload together with the UW sequence [9], [24]. Since our frame design in Sec. III does not consider the UW sequence within the FFT size, the payload block itself does not experience a circular channel condition. On the other hand, considering an odd size FFT not only increases the complexity, but also collects more noise samples in DFT domain i.e. $N$ time-domain noise samples instead of $N_{d}$. In the following, we derive a UW-free joint channel-equalization-and-demodulation for a generalized flexible waveform that can emulate any orthogonal or nonorthogonal single/multi-carrier. 


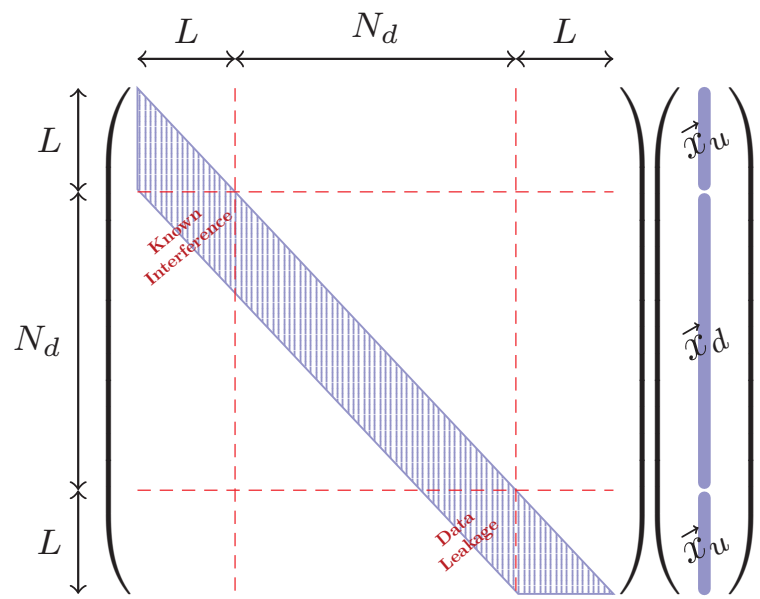

(a) Linear convolution

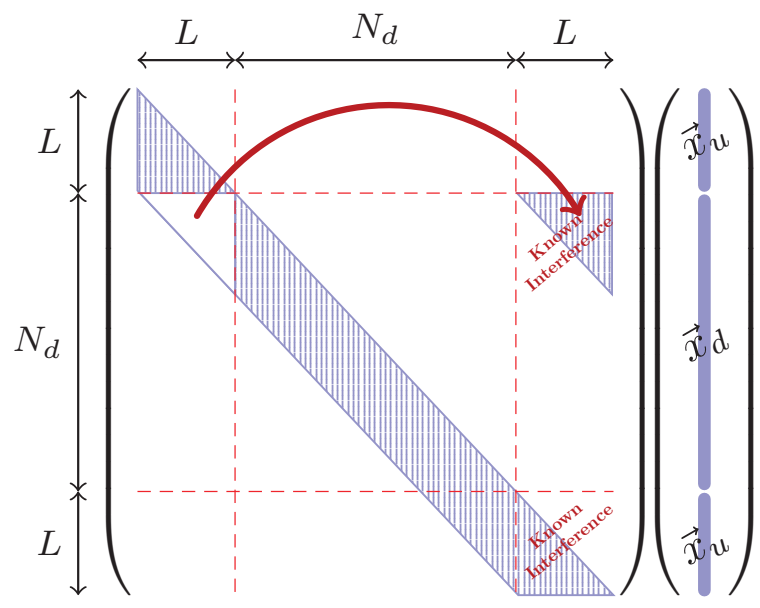

(c) Conventional UW-based systems

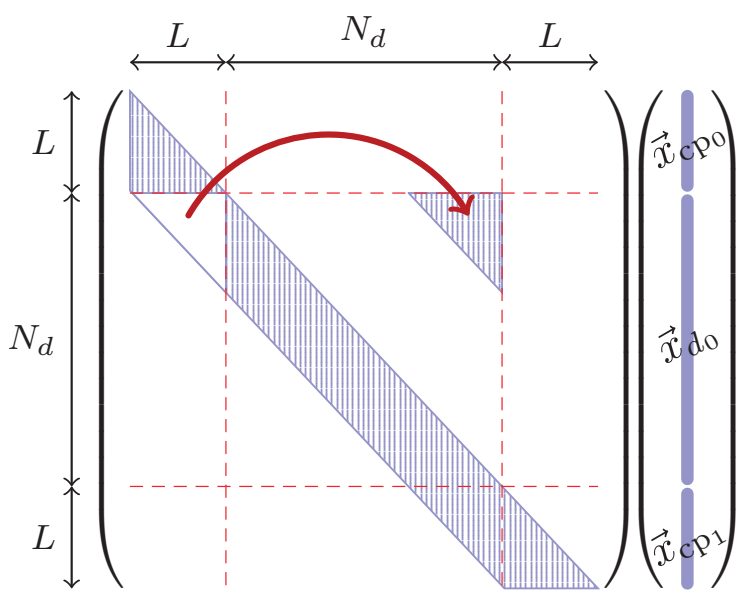

(b) CP-based system

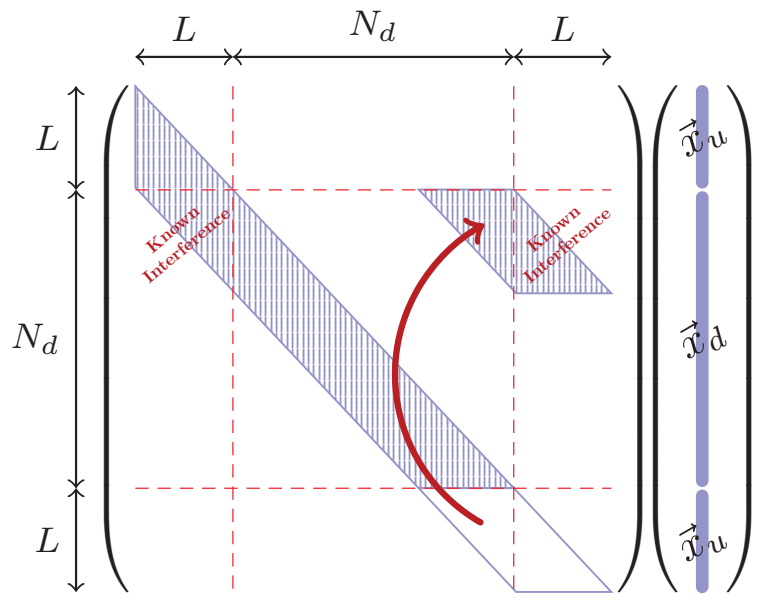

(d) UW-free approach

Fig. 5. The channel matrix $\mathcal{H}$ that different receiver types would experience in the linear model $\vec{y}=\mathcal{H} \vec{x}+\vec{w}$.

\begin{tabular}{|c|c|c|}
\hline UW & Payload & UW \\
\hline & $\overrightarrow{L-1}$ & $\overrightarrow{0: L-1}$ \\
\hline
\end{tabular}

Fig. 6. Emulating a circular CIR for the $N_{d}$ FFT-size payload sequence at the receiver.

\section{A. UW-free Equalization}

While in a block-fading system, the Tx signal experiences a linear convolution with the CIR, different techniques-as illustrated in Fig. 5-facilitate a cyclic signal observation at the receiver. In linear convolution, i.e. Fig. 5a, due to the $L$-tap CIR, the UW sequence transmitted before the payload causes some known interference ${ }^{4}$. Also due to the same reason, the last $L$ samples of $\vec{x}_{d}$ leak into the next UW sequence. The

${ }^{4}$ Given the knowledge of $\vec{x}_{u}$ and $\hat{\vec{h}}$, the UW influence can be removed at the receiver side. Although, due to the imperfect knowledge of $\hat{\vec{h}}$, we use the term known interference. From an estimation theory point of view, one shall refer the known interference as the mean of the signal, which must be removed before estimating $\vec{x}_{d}$. power of such data leakage would be wasted, if in a naive approach, one applies the FFT over $\vec{x}_{d}$. In Fig. 5b, one can see that in a CP-based system, since $\vec{x}_{\mathrm{cp}_{0}}$ is identical to the last $L$ samples of $\vec{x}_{d_{0}}$, the receiver can infer that $\vec{x}_{d}$ experiences a circular channel matrix. Similarly, in conventional UW-based systems (see Fig. 5c), the receiver can also emulate a cyclic signal condition for the transmitted $\vec{x}=\left[\vec{x}_{d}^{T}, \vec{x}_{u}^{T}\right]^{T}$ sequence. Though, the presence of $\vec{x}_{u}$ within the FFT block, causes known interference. For a UW-free equalization, i.e. Fig. 5d, we reconstruct a circular channel condition by adding the first $L$ samples of the UW to the first $L$ samples of the payload block as in Fig. 6. In this case, while the last $L$ samples of the payload has leaked into its following UW 
sequence, i.e. due to the linear convolution of the CIR with the $U W$-Payload- $U W$ sequence, taking the first $L$ samples of UW and adding it to the beginning of the payload sequence makes $\vec{y}_{\text {cyclic, } q} \in \mathbb{C}^{N_{d}}$ to experience a circular convolution of the transmitted payload sequence with the CIR. In this approach, $N_{d}+L \leq N$ noise samples are being collected, which is comparable to the $N$ noise samples collection of the conventional approach. However, due to the different noise variance at the first $L$ samples of the payload block, some noise correlation in frequency domain is to be expected.

Formally, we define the UW-free cyclic observed signal as

$$
\vec{y}_{\text {cyclic }, q} \triangleq \vec{y}_{d, q}+\left[\left(\vec{y}_{u, q}\right)_{[0: L-1]}^{T} \overrightarrow{0}_{N_{d}-L}^{T}\right]^{T},
$$

where $\left(\vec{y}_{u, q}\right)_{[0: L-1]}$ denotes the first $L$ samples of the received UW sequence after the payload, and $\vec{y}_{d, q} \in \mathbb{C}^{N_{d}}$ denotes the received payload sequence at block $b$. Further details concerning the observed signal $\vec{y}_{\text {cyclic }, q}$ can be found in the Appendix where we provide an algebraic proof that $\vec{y}_{\text {cyclic }, q}$ experiences a circular channel matrix. In (26) and the rest of expressions in this section, the subscript $b$ is ignored for brevity. Also note that in (26), the first $L$ samples of the $\vec{y}_{\text {cyclic, } q}$ are subject to noise variance $2 \sigma_{w}^{2}$, and therefore, if an $N_{d}$ point FFT size has been utilized, some noise correlation occurs in frequency domain. The above cyclic signal reconstruction approach provides the advantage of adapting radix-2 FFT-size for the payload size. Additionally, since the UW slot is no longer part of the FFT block, a low complexity equalization approach that relies on the structure of the modulation matrix $\mathcal{M}$ can be directly applied. For instance, an OFDM signal (i.e. $\mathcal{M}_{\text {ofdm }}=\boldsymbol{F}_{N_{d}}^{H}$ ) would have an almost diagonal effective channel, whereas, the circular pulse-shaping GFDM signal with $\mathcal{M}=A$ would have a banded matrix.

Once a cyclic receive signal via (26) has been obtained, we remove the UW influence and thus, the receive payload sequence in DFT domain yields (27) at the bottom of the page where $\hat{\boldsymbol{H}}_{q, i, N_{\mathrm{u}}}=\operatorname{diag}\left(\sqrt{N_{\mathrm{u}}} \boldsymbol{F}_{N_{\mathrm{u}}, L} \hat{\vec{h}}_{q, i}\right)$. In this case, the receive signal $\vec{Y}_{\text {cyclic }, d}=\left[\left(\vec{Y}_{\text {cyclic }, d, q}\right)_{q=1: \mathfrak{Q}}^{T}\right]^{T}$ can be decomposed into the linear model (28). Here $\overline{\mathcal{M}}=\boldsymbol{I}_{\mathfrak{I}} \otimes \mathcal{M}$, $\overline{\boldsymbol{F}}_{N_{d}}=\boldsymbol{I}_{\mathfrak{I}} \otimes \boldsymbol{F}_{N_{d}}, \overline{\overline{\boldsymbol{F}}}_{N_{d}}=\boldsymbol{I}_{\mathfrak{Q}} \otimes \boldsymbol{F}_{N_{d}}, \overline{\boldsymbol{F}}_{N_{\mathrm{u}}}=\boldsymbol{I}_{\mathfrak{I}} \otimes \boldsymbol{F}_{N_{\mathrm{u}}}$, $\vec{x}_{u}=\left[\left(\vec{x}_{u, i}\right)_{i=1: \mathfrak{I}}^{T}\right]^{T}, \vec{w}_{u} \in \mathbb{C}^{N_{\mathrm{u}}}$ denotes the AWGN process within the UW sequence, whereas $\vec{w}_{d} \in \mathbb{C}^{\mathfrak{Q} N_{d}}$ denotes the noise samples associated to the payload sequences of $\mathfrak{Q}$ antennas. Moreover, $\tilde{\boldsymbol{H}}_{N_{d}}=\boldsymbol{H}_{N_{d}}-\hat{\boldsymbol{H}}_{N_{d}}$ denotes the error of the channel estimation that is uncorrelated to $\vec{d}$. The matrix $\hat{\boldsymbol{H}}_{N_{d}} \in \mathbb{C}^{\mathfrak{Q} N_{d} \times \mathfrak{I} N_{d}}$ is MIMO version of $\mathfrak{Q} \times \mathfrak{I}$ matrices $\hat{\boldsymbol{H}}_{q, i, N_{d}}=\operatorname{diag}\left(\sqrt{N_{d}} \boldsymbol{F}_{N_{d}, L} \hat{\vec{h}}_{q, i}\right)$, (Likewise $\boldsymbol{H}_{N_{d}}, \tilde{\boldsymbol{H}}_{N_{d}}$ and $\tilde{\boldsymbol{H}}_{N_{\mathrm{u}}}$ with respective dimensions).

Considering the observation matrix $\hat{\boldsymbol{H}}_{N_{d}} \overline{\boldsymbol{F}}_{N_{d}} \overline{\mathcal{M}}$ in (28), the CWCU LMMSE [25] equalizer of $d$ with imperfect channel knowledge with covariance $\left(\widehat{\boldsymbol{\Sigma}}_{h h, \mathrm{~W}}\right)_{q, i}$-defined in (25)yields

$$
\begin{aligned}
\overrightarrow{\hat{d}} & =\frac{\boldsymbol{\Sigma}_{d Y}\left(\boldsymbol{\Sigma}_{d \hat{H}}+\boldsymbol{\Sigma}_{d \tilde{H}}+\boldsymbol{\Sigma}_{x_{u} \tilde{H}}+\boldsymbol{\Sigma}_{w_{u}}+\sigma_{w}^{2} \boldsymbol{I}_{\mathfrak{Q} N_{d}}\right)^{-1} \vec{Y}_{d}}{\operatorname{diag}^{-1}\left(\widetilde{\boldsymbol{\Sigma}}_{d d}\right)}, \\
\widehat{\boldsymbol{\Sigma}}_{d d} & =\boldsymbol{\Sigma}_{d d}-\widetilde{\boldsymbol{\Sigma}}_{d d},
\end{aligned}
$$

herein, we calculate each term as in (30a)-(30f) where $\boldsymbol{\Sigma}_{d d}=$ $\mathbb{E}\left[\vec{d} \vec{d}^{H}\right]=\boldsymbol{I}_{\mathfrak{I} N_{d}}$ provided that the elements of $\vec{d}$ are independent and identically distributed (i.i.d.) standard Gaussian random variables, $\widehat{\boldsymbol{\Sigma}}_{\mathrm{HH}, i}=\boldsymbol{F}_{N_{\mathrm{u}}, L}\left(\widehat{\boldsymbol{\Sigma}}_{h h, W}\right)_{q, i} \boldsymbol{F}_{N_{\mathrm{u}}, L}^{H}$ and for a centralized MIMO with identical PDP between Tx-Rx antenna pairs, $\widehat{\boldsymbol{\Sigma}}_{\mathrm{HH}, i}$ is identical for all $q \in\{1, \cdots, \mathfrak{Q}\}$. Further, $\boldsymbol{\Sigma}_{X_{u} X_{u}, i}=\boldsymbol{F}_{N_{\mathrm{u}}} \vec{x}_{u, i} \vec{x}_{u, i}^{H} \boldsymbol{F}_{N_{\mathrm{u}}}^{H}$. From a complexity point of view, the matrices $\boldsymbol{\Sigma}_{d \hat{H}}$ and $\boldsymbol{\Sigma}_{d \tilde{H}}$ become band diagonal matrices for GFDM with proper permutations. The lower and upper bandwidth of the two matrices is given by $B_{l}=B_{u}=\mathfrak{I}\left(M+N_{\alpha}\right)$, where $N_{\alpha}<M$ is the number of overlapping frequency bins with the neighboring subcarriers

$$
\begin{aligned}
& \vec{Y}_{\text {cyclic }, d, q}=\boldsymbol{F}_{N_{d}} \vec{y}_{\text {cyclic }, q}-\boldsymbol{F}_{N_{d}} \sum_{i=0}^{\mathfrak{I}}\left[\left(\boldsymbol{F}_{N_{\mathrm{u}}}^{H} \hat{\boldsymbol{H}}_{q, i, N_{\mathrm{u}}} \boldsymbol{F}_{N_{\mathrm{u}}} \vec{x}_{u, i}\right)_{[0: L-1]}^{T} \overrightarrow{0}_{N_{d}-L}^{T}\right]^{T}, \\
& \vec{Y}_{\text {cyclic }, d}=\hat{\boldsymbol{H}}_{N_{d}} \overline{\boldsymbol{F}}_{N_{d}} \overline{\mathcal{M}} \vec{d}+\tilde{\boldsymbol{H}}_{N_{d}} \overline{\boldsymbol{F}}_{N_{d}} \overline{\mathcal{M}} \vec{d} \\
& +\overline{\overline{\boldsymbol{F}}}_{N_{d}}\left[\left(\begin{array}{c}
\left.\left.\overline{\boldsymbol{F}}_{N_{\mathrm{u}}}^{H} \tilde{\boldsymbol{H}}_{N_{\mathrm{u}}} \overline{\boldsymbol{F}}_{N_{\mathrm{u}}} \vec{x}_{u}\right)_{0: L-1}\right)_{q=1: \mathfrak{Q}}^{T} \\
\hat{0}_{N_{d}-L}
\end{array}\right]^{T}+\overline{\overline{\boldsymbol{F}}}_{N_{d}}\left[\left(\begin{array}{c}
\left(\vec{w}_{u}\right)_{0: L-1} \\
\overrightarrow{0}_{N_{d}-L}
\end{array}\right)_{q=1: \mathfrak{Q}}^{T}\right]^{T}+\overline{\overline{\boldsymbol{F}}}_{N_{d}} \vec{w}_{d} .\right. \\
& \boldsymbol{\Sigma}_{d \hat{H}}=\hat{\boldsymbol{H}}_{N_{d}} \overline{\boldsymbol{F}}_{N_{d}} \overline{\mathcal{M}} \boldsymbol{\Sigma}_{d d} \overline{\mathcal{M}}^{H} \overline{\boldsymbol{F}}_{N_{d}}^{H} \hat{\boldsymbol{H}}_{N_{d}}^{H}, \\
& \boldsymbol{\Sigma}_{d \tilde{H}}=\boldsymbol{I}_{\mathfrak{Q}} \otimes \sum_{i=1}^{\mathfrak{I}} \boldsymbol{F}_{N_{d}} \mathcal{M} \boldsymbol{\Sigma}_{d d} \mathcal{M}^{H} \boldsymbol{F}_{N_{d}}^{H} \circ \widehat{\boldsymbol{\Sigma}}_{\mathrm{HH}, i}
\end{aligned}
$$

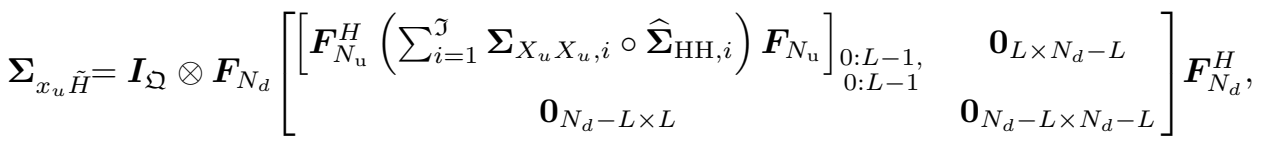

$$
\begin{aligned}
& \boldsymbol{\Sigma}_{w_{u}}=\boldsymbol{I}_{\mathfrak{Q}} \otimes \boldsymbol{F}_{N_{d}}\left[\begin{array}{cc}
\sigma_{w}^{2} \boldsymbol{I}_{L} & \mathbf{0}_{L \times N_{d}-L} \\
\mathbf{0}_{N_{d}-L \times L} & \mathbf{0}_{N_{d}-L \times N_{d}-L}
\end{array}\right] \boldsymbol{F}_{N_{d}}^{H}, \\
& \boldsymbol{\Sigma}_{d Y}=\boldsymbol{\Sigma}_{d d} \overline{\mathcal{M}}^{H} \overline{\boldsymbol{F}}_{N_{d}}^{H} \hat{\boldsymbol{H}}_{N_{d}}^{H} \text {, } \\
& \widetilde{\boldsymbol{\Sigma}}_{d d}=\boldsymbol{\Sigma}_{d Y}\left(\boldsymbol{\Sigma}_{d \hat{H}}+\boldsymbol{\Sigma}_{d \tilde{H}}+\boldsymbol{\Sigma}_{x_{u} \tilde{H}}+\boldsymbol{\Sigma}_{w_{u}}+\sigma_{w}^{2} \boldsymbol{I}_{\mathfrak{Q} N_{d}}\right)^{-1} \boldsymbol{\Sigma}_{d Y}^{H},
\end{aligned}
$$




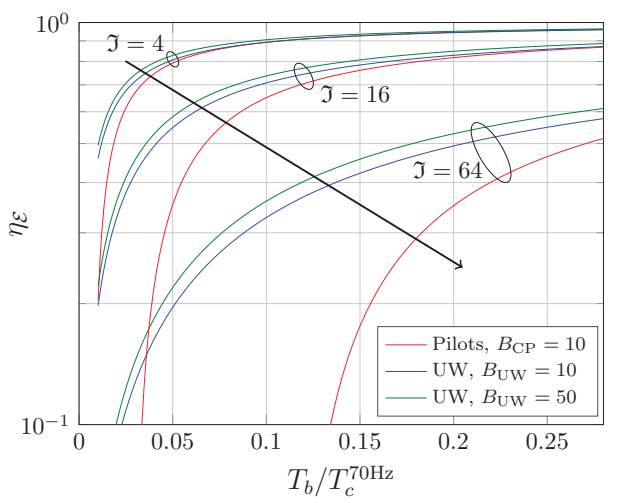

(a) ETU like channel, i.e. $L=9$.

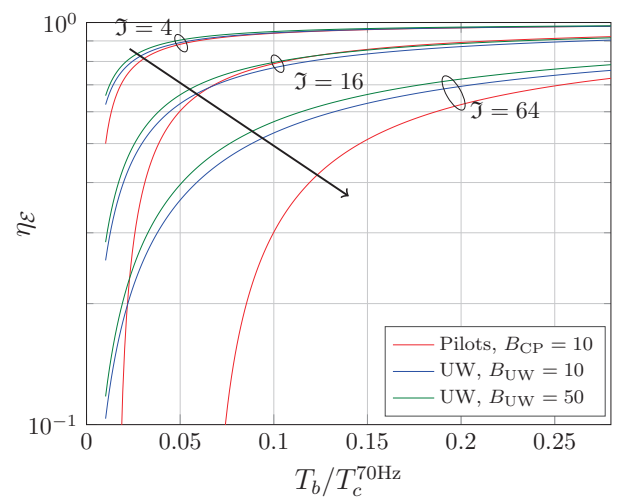

(b) EVA like channel, i.e. $L=5$.

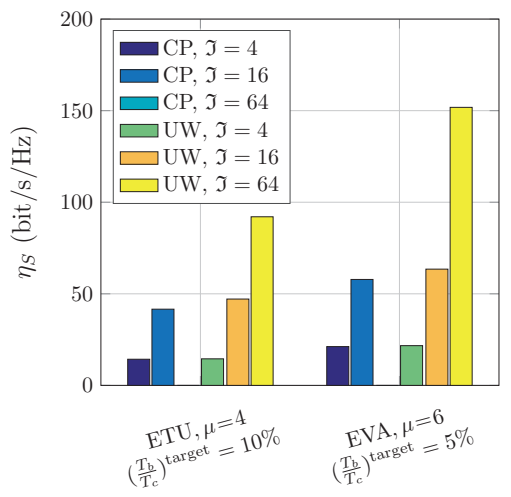

(c) Spectral efficiency.

Fig. 7. Energy and spectral efficiency of the systems for different number of Tx antennas $\mathfrak{I}$ while $T_{c}^{70 \mathrm{~Hz}} \approx 2.56 \mathrm{~ms}$.

and it depends on the filter roll-off factor $\alpha$. The matrix $\boldsymbol{\Sigma}_{x_{u} \tilde{H}}$ is a full $\mathfrak{Q} N_{d} \times \mathfrak{Q} N_{d}$ matrix which can be ignored by setting $\boldsymbol{\Sigma}_{x_{u} \tilde{H}}=\mathbf{0}_{\mathfrak{Q} N_{d} \times \mathfrak{Q} N_{d}}$. Moreover, $\boldsymbol{\Sigma}_{w_{u}}$ defined in (30d) shows that the noise becomes correlated in DFT domain and thus, for the sake of saving complexity, we consider taking its diagonal elements.

\section{NumERICAL AND Simulation RESUlts}

\section{A. Efficiency Analysis}

In this section, we make a numerical comparison of the energy and spectral efficiency of UW-based systems vs. CPbased systems in different MIMO setups. We assume that due to the mobility of the transceivers space-frequency pilots are necessary. The sampling frequency is set to $F_{s}=1.92 \mathrm{MHz}$ and the PDP is chosen to be similar to either long term evolution (LTE) extended typical urban (ETU) or extended vehicular A (EVA), i.e. $L_{\mathrm{ETU}}=9$ and $L_{\mathrm{EVA}}=5$ channel taps, which correspond to $4.7 \mu \mathrm{s}$ and $2.6 \mu \mathrm{s}$ maximum delay spread, respectively. The UW length $N_{\mathrm{u}}^{\mathrm{EVA-I}}$ for different antenna configurations and channel models is chosen to be $N_{\mathrm{u}}^{\mathrm{EVA}-4}=5^{2}, N_{\mathrm{u}}^{\mathrm{ETU}-4}=7^{2}, N_{\mathrm{u}}^{\mathrm{EVA}-16}=11^{2}, N_{\mathrm{u}}^{\mathrm{ETU}-16}=13^{2}$, $N_{\mathrm{u}}^{\mathrm{EVA}-64}=19^{2}, N_{\mathrm{u}}^{\mathrm{ETU}-64}=29^{2}$, and for the sake of primary synchronization a double length UW is used as preamble. In the CP-based system we also consider a preamble length of $N_{p}=2 N_{u}$ to be used for synchronization and afterwards, $B_{\mathrm{CP}}=10$ blocks are continuously being transmitted. The $\mathrm{CP}$ length is set to $N_{\mathrm{cp}}=L$ samples, and also $\mathfrak{I} L$ orthogonal pilots are inserted into every block.

Fig. 7 compares the energy and spectral efficiency of the UW- vs. CP-based systems for two channel models and different number of Tx antennas. Here, we consider an intermediate mobility condition with maximum Doppler shift $f_{d}=70 \mathrm{~Hz}$ and thus, the coherence time becomes $T_{c}^{70 \mathrm{~Hz}} \approx \frac{9}{16 \pi f_{d}}=$ $2.56 \mathrm{~ms}$. In order to design the transmit blocks such that they experience a near block-fading conditions, the block duration $T_{b}=N_{d} / F_{s}$ is commonly chosen to be smaller than $10 \%$ of the coherence time. Thus, in Fig. 7, we divide the $\mathrm{x}$-axis by $T_{c}$ in order to clearly find the corresponding target ${ }^{5} \frac{T_{b}}{T_{c}}$. As can be seen in Fig. 7, the energy efficiency of the UWbased systems with $B_{\mathrm{UW}}=10$ and $B_{\mathrm{UW}}=50$ is always larger than its corresponding CP-based system for short block duration, because the total resource size in UW-based systems is larger (see Sec. III-B). On the other hand, if the coherence time $T_{c}$ becomes larger, also larger block duration $T_{b}$ can be chosen, and in that case, the efficiency curves of the UW- vs. CP-based systems tend to merge. We also note that in each channel scenario, by increasing the number of Tx. antennas $\mathfrak{I}$, the energy efficiency of the systems decreases. Indeed, large number of Tx. antennas yield in increase of the channel parameters that need to be estimated, and therefore, more resources must be dedicated to reference signals (either UW or pilots) to estimate the $\Im L$ parameters. Although, comparing ETU and EVA like channels, $\eta_{\mathcal{E}}$ decreases with a slower pace for EVA, due to the smaller channel length $L$. The spectral efficiency of the MIMO systems has been depicted in Fig. 7c where we have assumed that the data is spatially multiplexed over all Tx antennas. Here, assuming a very selective channel, i.e. ETU, and $10 \%$ target $\frac{T_{b}}{T_{c}}$, i.e. channel varies slightly within the block, we choose 16-QAM, i.e. $\mu=4$, as a sufficiently robust modulation order to decode the data. On the other hand, for an EVA like channel, we set the target $\frac{T_{b}}{T_{c}}$ to $5 \%$ in order to achieve near block-fading conditions, although, smaller $\frac{T_{b}}{T_{c}}$ yields increase of overhead. In this case, as the channel is less selective and nearly block-fading, choosing higher modulation order, e.g. $\mu=6$ becomes feasible. Note that the $10 \%$ and $5 \%$ $\left(\frac{T_{b}}{T_{c}}\right)^{\text {target }}$ correspond to 0.1 and $0.05 T_{b} / T_{c}{ }^{70 \mathrm{~Hz}}$ in figures $7 \mathrm{a}$ and $7 \mathrm{~b}$, respectively. As we observe in Fig 7c, although the energy efficiency of the systems decreases with increase of Tx antennas, the spectral efficiency increases rapidly for the UWbased systems. Nonetheless, such condition is not necessarily true for a CP-based system, because, for $\mathfrak{I}=64$ and short block duration $T_{b}$, the number of subcarriers are not sufficient for estimating all the channel parameters, and thus $\eta_{S \text {,Pilots }}$ for the chosen $\left(\frac{T_{b}}{T_{c}}\right)^{\text {target }}$ and $\mathfrak{I}=64$ becomes zero. We also note

\footnotetext{
${ }^{5}$ As a matter of fact, if the ratio $\frac{T_{b}}{T_{c}}$ is large, the channel varies too quickly within the block duration, and consequently, due to the channel estimation and equalization imperfections, it limits the choice of modulation order $\mu$.
} 
that in Fig. 7c, although $\left(\frac{T_{b}}{T_{c}}\right)^{\text {target }}$ in EVA channel is chosen to be smaller than that of ETU channel, the spectral efficiency still increases due to the larger modulation order $\mu$.

\section{B. $O O B$ Emission}

The performance of the UW-based GFDM in terms of OOB radiation is depicted in Fig. 8. Here, the GFDM waveform is configured to have $K_{\text {on }}=22$ active subcarriers out of $K=64$ subcarriers, and a raised-cosine filter [26] with roll-off factor $\alpha=0.3$ has been chosen for it. The total number of subsymbols is set to $M=16$ and all $M$ subsymbols are active for the basic GFDM as well as UW-GFDM. Besides, UW-GS-GFDM and GS-GFDM use $M_{\text {on }}=15$ active subsymbols while their first subsymbol is configured as a guard symbol according to the description of Sec. IV-A. Moreover, the UW sequence is initially obtained via (13) by letting $N_{\mathrm{u}}=K_{\mathrm{on}}^{\mathrm{UW}}=25$ and afterwards, it is zero-padded to the total number of $K^{\mathrm{UW}}=72$ subcarriers. The power spectral density (PSD) of a CP-OFDM signal with the same number of subcarriers and UW-DFTs-OFDM based on the approach of [6] are also plotted as benchmarks. Here, the UW sequences in DFT-s-OFDM are initially generated in form of PN-sequence that are mapped to QPSK symbols and then circularly filtered by a sinc function. Note that the comparison with UW-DFT-s-OFDM remains valid for only SISO systems ${ }^{6}$.

As can be seen in Fig. 8, UW-GFDM without a guard symbol increases the OOB emission of basic GFDM which is due to the abrupt changes of the signal at the boundary of UW sequence and the GFDM signal. Setting the first GFDM subsymbol as a GS, we observe that OOB radiation of UW-GS-GFDM reduces by an order of magnitude. However, UW-GS-GFDM still suffers from higher OOB emission when it is compared to GS-GFDM, which is due to the abrupt changes of the signal within the UW sequence. We also note that at frequency of around $\frac{f}{F_{s}} \approx 31$, there is a peak of energy for UW-GS-GFDM and GS-GFDM which is due to the $\delta\left[k-k_{p}\right]$ function with energy $\sqrt{K}$. Comparing the UWGS-GFDM with UW-DFT-s-OFDM, we observe that DFT-sOFDM achieves a slightly smaller OOB radiation due to the frequency domain filtering of the UW sequence, whereas the UW-GS-GFDM employs the frequency domain zero-padded version of the sequence (13) without filtering. We also note that the PSD curve of UW-DFT-s-OFDM has an asymmetrical shape because of the employed PN-sequence.

\section{Channel Estimation}

Consider a $4 \times 4$ MIMO system over an ETU-like channel with $L=9$ taps of PDP from 0 to $-20 \mathrm{~dB}$. Sampling frequency is set to $F_{s}=1.92 \mathrm{MHz}$, UW length $N_{u}=49$ and payload length $N_{d}=256$. We also consider $B=50$ blocks after preamble to further emphasize the influence of WienerHopf filters. The time-varying channel estimation is simulated

\footnotetext{
${ }^{6}$ As the UW-DFT-s-OFDM signal is jointly generated by precoding the data and filtering deterministic QPSK symbols, the resulting UW sequence does not provide the optimum cross-correlation properties for a high quality MIMO synchronization. In addition, as the UW-DFT-s-OFDM signal based on timedomain QPSK symbols is not constant magnitude in frequency domain, they are not optimum for MIMO channel estimation either.
}

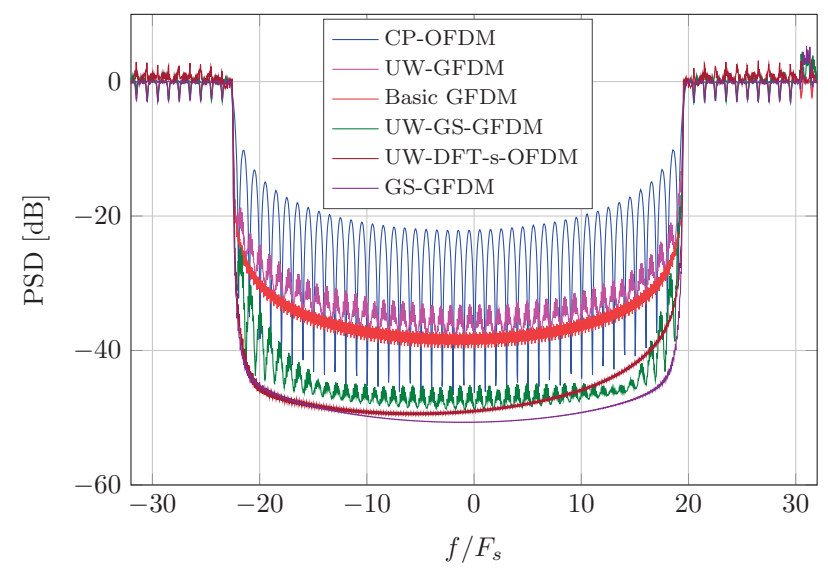

Fig. 8. Out of band radiation.

by considering that each tap of the CIR being temporally correlated according to [27] $\left[\mathbf{R}_{h}\right]_{n, n^{\prime}}=J_{0}\left(2 \pi\left(n-n^{\prime}\right) \nu_{d}\right)$, where $\nu_{d}=f_{d} / F_{s}$ and $J_{0}(\cdot)$ denotes the zeroth order Bessel function of the first kind. The number of observations of the UW sequences would be $N_{u}-L=40$ time domain samples (i.e. $\vec{y}_{p, q} \in \mathbb{C}^{40}$ ). The CP-OFDM system is configured to adopt $N_{\text {ofdm }}^{\mathrm{ON}}=240$ active subcarriers out of $N_{\text {ofdm }}=256$, in which 40 equispaced of them are pilot subcarriers. The OFDM pilot subcarriers are being generated via $\mathrm{PN}$-sequences mapped into QPSK constellations. The MSE of the channel estimation is calculated by comparing the DFT domain of the estimated channel responses with respect to the true channel realizations at the center of each payload block.

Fig. 9 shows the MSE of the channel estimation for the proposed UW-based frames vs. CP-OFDM system. Comparing the pilot-aided CE of CP-OFDM with blockwise UW-based CE, i.e. (19), at $f_{d}=300 \mathrm{~Hz}$, we observe that at low SNR the performances are the same, however, at high SNR values CPOFDM pilots suffer from nearly one order of magnitude larger error floor which is due to the channel variations within the OFDM block duration. On the other hand, Blockwise UWbased CE at $f_{d}=300$ follows its $f_{d}=0$ bound with a very close gap for SNR values $<25 \mathrm{~dB}$, and at high SNR values, it has a smaller error floor than the CP-OFDM pilots, because the number of channel realizations within the UW sequence is smaller than that of CP-OFDM block duration (i.e. $N_{u}-L=40$ channel realizations vs. $N_{\text {ofdm }}=256$ for CP-OFDM). In other words, the energy of the UW sequences are concentrated into $\left(N_{u}-L\right)$-length time slot (i.e. $3.5 \%$ of the channel coherence time), whilst in CP-OFDM, the energy of the pilots are distributed over $N_{\text {ofdm }}=256$ samples (i.e. $22.3 \%$ of the channel coherence time). Furthermore, in CP-OFDM the $N_{\text {ofdm }}$ samples of the pilot subcarriers are contaminated by the inter-carrier-interference (ICI) from data subcarriers in such a time-varying channel condition. Comparing the blockwise UW-based channel estimations with their corresponding Wiener-filtered cases, i.e. (23), one may note that at low-mobility, i.e. $f_{d}=0$, the Wiener filter provides nearly $5 \mathrm{~dB}$ gain with respect to its blockwise channel estimation at $\mathrm{MSE}=10^{-3}$, although, at high mobility scenario, i.e. $f_{d}=300$, the gain is marginal, because the matrix $\mathbf{R}_{h}$ 


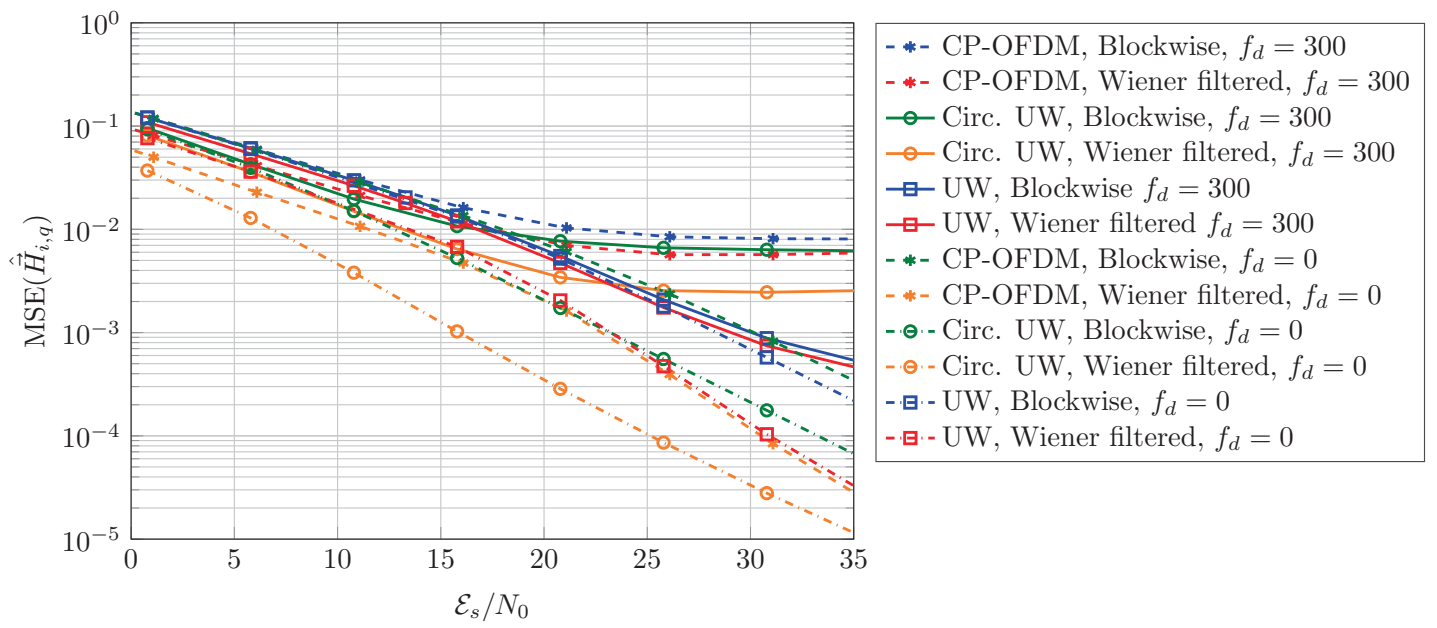

Fig. 9. Channel Estimation Performance.

defined in (22) considers less temporal correlation of the channel estimations. Moving on to the channel estimation performances of Circ.-UW sequences, we observe that at lowmobility cases, the blockwise Circ.-UW CE outperforms the normal UW-based CE as well as blockwise CP-OFDM with nearly 5 and $6.6 \mathrm{~dB}$ gain, respectively, whereas, the Wienerfiltered Circ.-UW gains more than $7.3 \mathrm{~dB}$ with respect to its normal Wiener-filtered UW-based CE at $f_{d}=0$. The reason for such performance gain shall be explained by comparing the condition number of the observation matrices

$$
\begin{aligned}
\operatorname{Cond}\left(\boldsymbol{X}_{\text {obs }}^{\text {circ.UW }}\right)= & 4.54 \\
<\operatorname{Cond}( & \left.\boldsymbol{X}_{p}\right)=13.4 \\
& <\operatorname{Cond}\left(\boldsymbol{X}_{p}^{\text {OFDM pilots }}\right)=33.78 .
\end{aligned}
$$

Observing the performance of Circ.-UW-based channel estimation at high mobility $f_{d}=300$, the error floor at high SNR values increases with respect to the normal UW-based CE because of the same reason that happens to CP-OFDM, i.e. the two UW sequences observe $2\left(N_{u}-L\right)=80$ channel realizations. The Wiener-filtered Circ.-UW-based CE at $f_{d}=300$ does not provide significant gain at low SNR values (due to small temporal correlation of the estimates), whereas, at high SNR values, it reduces the channel estimation error floor.

\section{Equalization}

Given the imperfect channel estimation techniques proposed in Sec. V, we evaluate the transceiver performances in terms of uncoded symbol error rate (SER), mutual information (MI), and coded frame error rate (FER) via Monte Carlo simulations. Here, we consider again the same simulation parameters of Sec. VII-C and on top, we consider that the transmitted bits are encoded via Parallel Concatenated Convolutional Codes with code-rate $r_{\text {code }}=2 / 3$ and octal generator polynomial $(1,15 / 13)$, and they are mapped into 16-QAM symbols, i.e. modulation order $\mu=4$. The receiver employs the CWCU LMMSE equalization technique and transforms the equalized signal constellations into maximum likelihood (ML) log-likelihoods by taking into account the fact that each element of the received constellations $\overrightarrow{\hat{d}}=\vec{d}+\overrightarrow{\tilde{w}}$ subjects to an effective noise enhancement approximately distributed as $\overrightarrow{\tilde{w}} \sim \mathcal{N}_{C}\left(\overrightarrow{0}_{N_{d}}, \operatorname{diag}\left(\frac{1}{\operatorname{diag}^{-1}\left(\widetilde{\boldsymbol{\Sigma}}_{d d}\right)}\right)-\sigma_{w}^{2} \overrightarrow{1}_{N_{d}}\right)$ [28]. The SNR per bit is denoted by $E_{b} / N_{0}$ and it includes the gain of modulation and coding (i.e. $\mathcal{E}_{b} / N_{0}=\mathcal{E}_{s} / N_{0}-10 \log _{10} \mu r_{\text {code }}$, where $\mathcal{E}_{s} / N_{0}$ denotes SNR per symbol). As benchmarks, in addition to the CP-OFDM pilot transmission (i.e. identically configured as in Sec. VII-C), we also consider a conventional approach of UW-based transmission for GFDM similar to a MIMO extended version of [9], i.e. the channel estimation of UW slot $b-1$ is used for its following payload block $b$, and also the estimated channel matrix $\hat{\boldsymbol{H}}$ and the GFDM modulation matrix $\boldsymbol{A}$ are being separately equalized via LMMSE approach and without considering the imperfect CE statistics (i.e. setting $\boldsymbol{\Sigma}_{d \tilde{H}}=\boldsymbol{0}_{\mathfrak{Q} N_{d} \times \mathfrak{Q} N_{d}}$ and $\left.\boldsymbol{\Sigma}_{x_{u} \tilde{H}}=\mathbf{0}_{\mathfrak{Q} N_{d} \times \mathfrak{Q} N_{d}}\right)$. Moreover, the conventional approach uses the circularity of the channel over the Payload- $U W$ time slot and thus, calculation of $\boldsymbol{\Sigma}_{w_{u}}$ is also unnecessary.

Fig. 10a compares the SER performance of the proposed approaches with the state-of-the-art equalization techniques at high mobility scenarios. One may notice that the conventional UW-based EQ. for GFDM suffers from a large error floor in SER performance due to three major aspects: a) The GFDM signal suffers from self-interference caused by the circular filtering, b) the conventional approach uses the $\mathrm{CE}$ of a single UW before the payload sequence, and c) it does not consider the imperfect CE statistics. The CP-OFDM also suffers from large error floor of SER, because at high mobility its channel estimation has the largest error floor, and also the orthogonality of the symbols are being lost due to the ICI caused by Doppler effects. Comparing the performance of UW-GFDM with Circ.UW-GFDM, one may notice that at high mobility of $f_{d}=300$, Circ.-UW-GFDM with blockwise CE approach has larger error floor than normal UW-GFDM with blockwise CE, because its observation considers a longer time duration with respect to the channel coherence time than the normal UW-GFDM. But on the contrary, the Wiener filtering that has a marginal gain in normal UW-GFDM, achieves a better performance gain in Circ.-UW-GFDM. Comparing the performances of CP- 


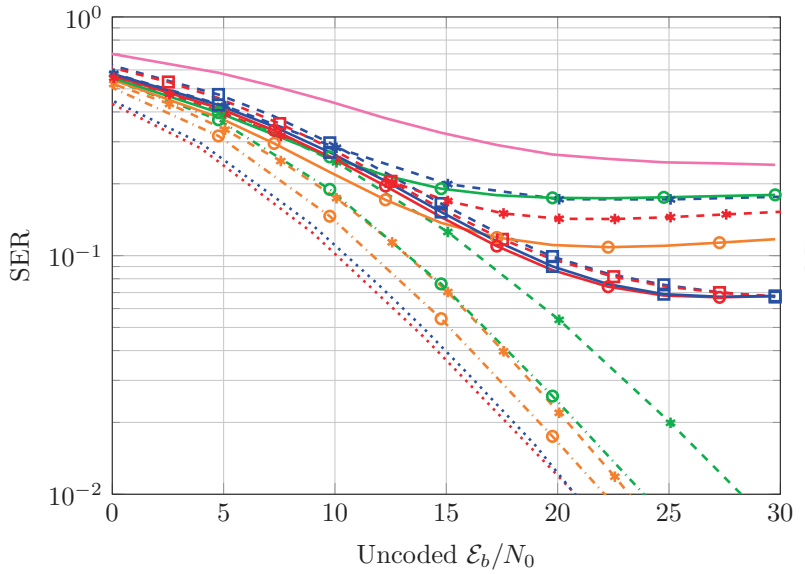

(a) Uncoded SER for 16-QAM symbols

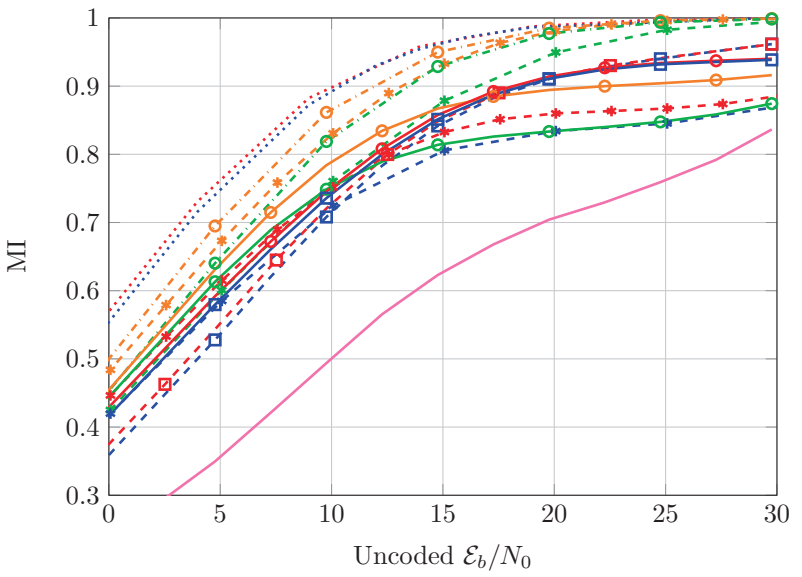

(b) MI of Tx bits and Rx bit LLRs

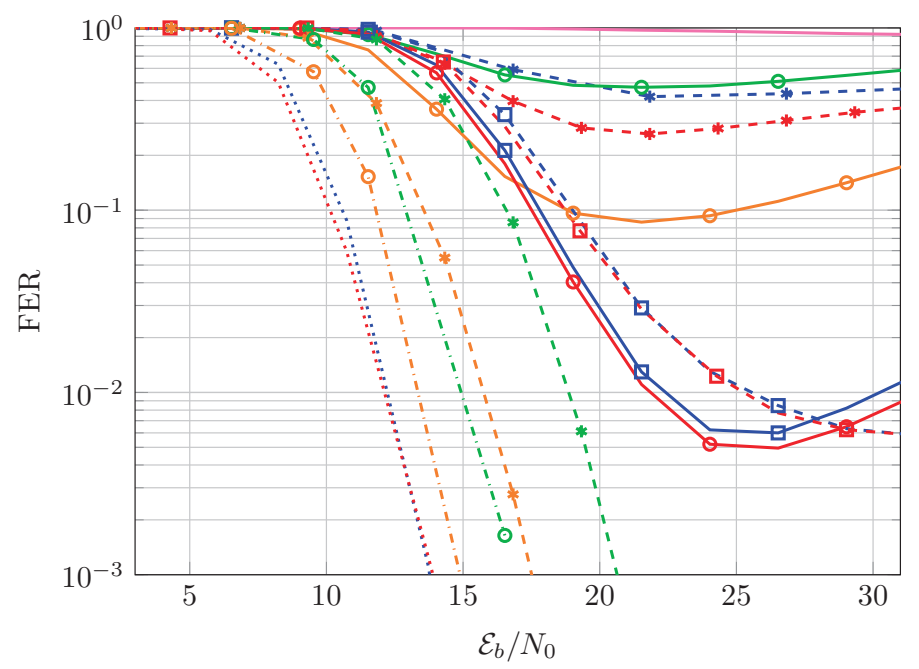

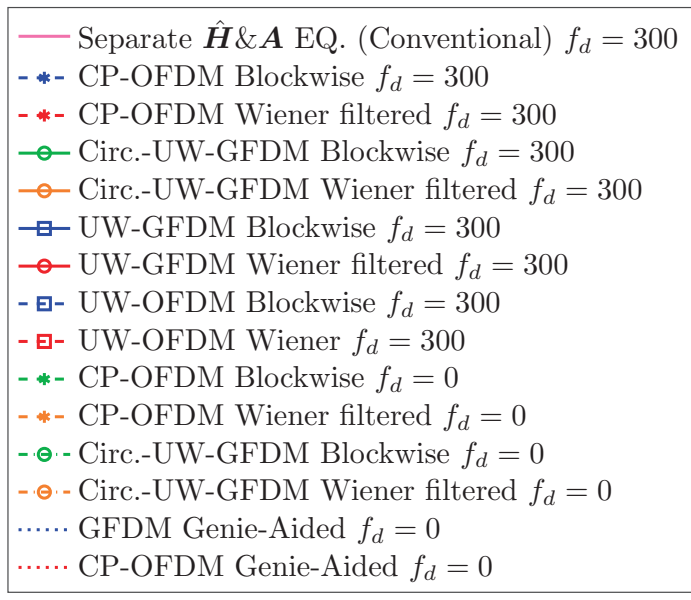

(c) Coded performance of 16-QAM 2/3 turbo codes with soft demapping.

Fig. 10. Equalization and Detection Performance of MIMO UW-based systems.

OFDM vs. Circ.-UW-GFDM at stationary channel conditions, i.e. $f_{d}=0$, we note that Circ.-UW-GFDM with blockwise CE outperforms the blockwise CP-OFDM by almost $3 \mathrm{~dB}$ SNR, which is mainly due to the better channel estimation of Circ.-UW-GFDM. Applying Wiener filter on top of them, the gap becomes smaller, although, the Wiener filtered Circ.UW-GFDM achieves a SER performance that is closest to its Genie-Aided receiver ${ }^{7}$. Note that here the gap between SER of Circ.-UW-GFDM and Genie-Aided receiver is $\sim 1.19 \mathrm{~dB}$, in which $\sim 0.79 \mathrm{~dB}$ is due to the $\mathrm{UW}$ overhead. Comparing the GFDM and OFDM systems with equal channel state information, we observe that Genie-aided receiver of OFDM has a marginal gain compared to its equivalent GFDM system, however, for the receivers with imperfect channel knowledge, i.e. UW-GFDM and UW-OFDM, the frequency diversity is better exploited in GFDM with its wider subcarriers. For further details of GFDM vs. OFDM systems with identical perfect and imperfect channel knowledge, we refer the interested readers to [28].

\footnotetext{
${ }^{7}$ Genie-Aided receiver considers the perfect channel knowledge without the need of UW transmission.
}

The demapper performances in terms of mutual information of transmitted bits (after encoder) and received bit LLRs (before decoder) are compared in Fig. 10b. As could be expected, the MI curves of different systems follow a similar behavior of their SER performances. For instance, the Wienerfiltered Circ.-UW-GFDM at block-fading situation achieves a higher MI than the Wiener-filtered CP-OFDM for the same reason that occurred to its SER performance. In addition, an interesting observation from Fig. 10b is the behavior of the curves for interference-limited systems. Here, we note that the MI curves of conventional UW-GFDM, CP-OFDM, blockwise and Wiener-filtered Circ.-UW-GFDM at $f_{d}=300 \mathrm{~Hz}$ tend to saturate over a nearly horizontal line between SNR values of 15 and $25 \mathrm{~dB}$. However, at very high SNR values, the MI curves start to increase again. We explain this behavior by considering the fact that the interference-statistics occurring due to the time-varying channels have not been considered in computation of (30f). Therefore, as the input SNR of the demapper increases, the output bit LLRs have higher confidence and thus, they result in higher mutual information. Nevertheless, as we see in the following, such high confident 
LLRs do not necessarily yield correct decoding performance.

Observing the detection performances in terms of FER, i.e. Fig. 10c, one can see that the FER performances of different EQ. techniques follow a similar behavior that already has been seen in SER and MI performances. However, at very high $\mathcal{E}_{b} / N_{0}$ values, we observe that all high mobility curves tend an upward trend, which happens due to the ICI (caused by Doppler and GFDM's self-interference) that has not been considered by the CWCU-LMMSE EQ. and the decoder. As also explained in the MI performances, by ignoring the offdiagonal elements of $\widetilde{\boldsymbol{\Sigma}}_{d d}$ and also the interference-statistics due to time-varying channels, the bit LLRs become too much confident, which results to have a worse FER at very high $\mathcal{E}_{b} / N_{0}$ values. Such an upward trend of the curves shall be solved by employing a time-varying equalizer, and/or an iterative MMSE parallel-interference-cancellation detection technique, e.g. [28].

\section{CONCLUSION}

In this paper, we have proposed a new UW-based MIMO frame design for multi-carrier transmission over frequency selective and time-variant channels. Through energy and spectral efficiency analysis, we have shown that if large number of antennas are employed at the transmitter, the proposed UW-based frame design achieves a significantly higher spectral efficiency than the conventional pilot-aided CP-OFDM systems. Employing orthogonal UW sequences for a non-orthogonal MIMO system, the proposed UW-GFDM maintains the spectral properties of CP-GFDM with slight degradation. In addition, via derivations of the LMMSE-based channel estimation and their corresponding Wiener-Hopf filters, we have observed that the proposed UW-based frame design achieves nearly one order of magnitude higher estimation accuracy in comparison to the pilot-aided CP-OFDM system. Providing an analytical proof for the proposed UW-free equalization technique, we have shown that the UW sequences does not necessarily need to be placed within the FFT-size and therefore, no tail-suppressionprecoder is required at the transmitter side. In the future, we would be interested in taking advantage of per-sample based channel estimation of the Wiener-Hopf filter, and apply a low complexity, time-varying channel equalization for the MIMO non-orthogonal multi-carriers.

\section{APPENDIX}

\section{Proof THAT (26) EMUlates A CIRCULAR CIR AT THE RECEIVER}

In this Appendix, we mathematically prove that the definition of $\vec{y}_{\text {cyclic, } q}$ in (26) emulates a circular channel matrix for the receive signal. Without loss of generality, as the extension of SISO to MIMO in this proof is a straightforward approach, we ignore the antenna indexes $i$ and $q$ for brevity. Additionally, we set the UW length to $L$ samples (i.e. $\vec{x}_{u} \in \mathbb{C}^{L}$ ) for simplicity of the expressions. By extending the vector-matrix dimensions in a respectful manner, the proof also remains valid for the cases where the length of $\vec{x}_{u}$ is larger than $L$.
Consider a stationary channel condition where the channel matrix based on linear convolution is a lower triangular Toeplitz matrix decomposed as follows:

$$
\boldsymbol{T} \triangleq\left[\begin{array}{ccc}
\mathcal{T}_{\mathbf{1 1}} & \mathbf{0}_{L \times N_{d}} & \mathbf{0}_{L \times L} \\
\mathcal{T}_{\mathbf{2 1}} & \mathcal{T}_{\mathbf{2 2}} & \mathbf{0}_{N_{d} \times L} \\
\mathbf{0}_{L \times L} & \mathcal{T}_{\mathbf{3 2}} & \mathcal{T}_{\mathbf{3 3}}
\end{array}\right]
$$

where $\mathcal{T}_{11} \in \mathbb{C}^{L \times L}, \mathcal{T}_{\mathbf{2 2}}=\left[\begin{array}{cc}\mathcal{T}_{\mathbf{2 2 , 1 2}} & \mathbf{0}_{L \times L} \\ \mathcal{T}_{\mathbf{2 2 , 2 1}} & \mathcal{T}_{\mathbf{2 2 , 2 2}}\end{array}\right] \in$ $\mathbb{C}^{N_{d} \times N_{d}}$ and $\mathcal{T}_{33} \in \mathbb{C}^{L \times L}$ are also lower triangular Toeplitz matrices with the CIR $\vec{h} \in \mathbb{C}^{L}$ on their first column. Moreover, the matrix $\mathcal{T}_{\mathbf{2 2 , 1 2}}$ takes only the first $L$ rows and the first $N_{d}-L$ columns of $\boldsymbol{T}_{\mathbf{2 2}} ; \mathcal{T}_{\mathbf{2 2 , 2 1}}$ contains the last $N_{d}-L$ rows and the first $N_{d}-L$ columns of $\mathcal{T}_{\mathbf{2 2}}$ and $\mathcal{T}_{\mathbf{2 2 , 2 2}}$ includes the last $N_{d}-L$ rows and the last $L$ columns of $\mathcal{T}_{\mathbf{2 2}}$. For the receive signal

$$
\vec{y}_{\text {UW-P-UW }}=\mathcal{T}\left[\vec{x}_{u}^{T}, \vec{x}_{d}^{T}, \vec{x}_{u}^{T}\right]^{T}+\vec{w}_{\text {UW-P-UW }},
$$

the matrices $\mathcal{T}_{\mathbf{2 1}}=\left[\begin{array}{ll}\mathcal{T}_{\mathbf{2 1}, \mathbf{1}}^{T}, & \mathbf{0}_{\left(N_{d}-L\right) \times L}^{T}\end{array}\right]^{T}$ and $\mathcal{T}_{\mathbf{3 2}}=$ $\left[\mathbf{0}_{L \times\left(N_{d}-L\right)}, \mathcal{T}_{\mathbf{3 2 , 2}}\right]$ cause the leakage of the first UW sequence into the receive counterpart of $\vec{x}_{d}$ and the leakage of the payload into the receive counterpart of second UW sequence, respectively. Here, $\mathcal{T}_{\mathbf{2 1 , 1}}=\mathcal{T}_{\mathbf{3 2 , 2}} \in \mathbb{C}^{L \times L}$ are identical upper triangular Toeplitz matrices with $\left[\vec{h}_{(1: L-1)}^{T}, 0\right]^{T}$ on their last column.

We note that in a CP-based system where the left $\vec{x}_{u}$ in (32) is substituted by $\vec{x}_{\mathrm{cp}}$ (i.e. the last $L$ samples of $\vec{x}_{d}$ ), the receive payload $\vec{y}_{d, \text { CP-based }}=\mathcal{C}_{\mathrm{CP} \text {-based }} \vec{x}_{d}+\vec{w}_{d}$ experiences a circular channel $\mathcal{C}_{\text {CP-based }}=\left[\begin{array}{cc}\mathcal{T}_{22,12} & \mathcal{T}_{21,1} \\ \mathcal{T}_{22,21} & \mathcal{T}_{22,22}\end{array}\right]$. In fact, having the matrix $\mathcal{T}_{\mathbf{2 1 , 1}}$ on top right corner of $\mathcal{T}_{\mathbf{2 2}}$ makes the matrix $\mathcal{C}_{\mathrm{CP} \text {-based }}$ circulant. In conventional UW-based systems where the circularity of the channel is facilitated by taking an $N$-point FFT over Payload-UW block, we have $\vec{y}_{\mathrm{P}-\mathrm{UW}}=\mathcal{C}_{\text {conv-UW }}\left[\vec{x}_{d}^{T}, \vec{x}_{u}^{T}\right]^{T}+\vec{w}_{\mathrm{P}-\mathrm{UW}}$, where $\mathcal{C}_{\text {conv-UW }}=$ $\left[\begin{array}{ll}\mathcal{T}_{22} & \mathcal{T}_{21} \\ \mathcal{T}_{32} & \mathcal{T}_{33}\end{array}\right]$ and thus, $\mathcal{C}_{\text {conv-Uw }}$ is also ciruclant. In (26) however, we aim to generate a circulant channel $\mathcal{C}$ over only the payload sequence, and thus obtain the receive signal

$$
\vec{y}_{d}=\mathcal{H} \vec{x}_{d}+\vec{w}_{d}^{\prime}
$$

In order to prove that $\mathcal{H}=\mathcal{C}$, we decompose the receive Payload-UW signal as

$$
\left[\begin{array}{l}
\vec{y}_{d} \\
\vec{y}_{u}
\end{array}\right]=\mathfrak{C}\left(\mathcal{T} \mathfrak{R}\left[\vec{x}_{d}^{T}, \vec{x}_{u}^{T}\right]^{T}+\left[\begin{array}{c}
\vec{w}_{d} \\
\vec{w}_{u}
\end{array}\right]\right),
$$

where each individual matrix is defined as in (34a) and (34b) at the top of next page.

Here, the repetition matrix $\mathfrak{R}$ is located at the transmitter side and it copies the UW sequence before the payload, i.e. $\mathfrak{R}\left[\vec{x}_{d}^{T}, \vec{x}_{u}^{T}\right]^{T}=\left[\vec{x}_{u}^{T}, \vec{x}_{d}^{T}, \vec{x}_{u}^{T}\right]^{T}$. On the other hand, the matrix $\mathfrak{C}$, which is located at the receiver side performs the operation defined in (26).

Evaluating the effective channel of $\left[\vec{x}_{d}^{T}, \vec{x}_{u}^{T}\right]^{T}$ in (34), we have (35) where

$$
\mathcal{C}=\left[\begin{array}{cc}
\mathcal{T}_{22,12} & \mathcal{T}_{32,2} \\
\mathcal{T}_{22,21} & \mathcal{T}_{22,22}
\end{array}\right]=\mathcal{H}
$$




$$
\begin{aligned}
& \mathfrak{R} \triangleq\left[\begin{array}{cc}
\mathbf{0}_{L \times N_{d}} & \boldsymbol{I}_{L} \\
\boldsymbol{I}_{N_{d}} & \mathbf{0}_{N_{d} \times L} \\
\mathbf{0}_{L \times N_{d}} & \boldsymbol{I}_{L}
\end{array}\right] \\
& \mathfrak{C} \triangleq\left[\begin{array}{cccc}
\boldsymbol{0}_{L \times L} & \boldsymbol{I}_{L} & \mathbf{0}_{L \times\left(N_{d}-L\right)} & \boldsymbol{I}_{L} \\
\mathbf{0}_{\left(N_{d}-L\right) \times L} & \mathbf{0}_{\left(N_{d}-L\right) \times L} & \boldsymbol{I}_{N_{d}-L} & \mathbf{0}_{\left(N_{d}-L\right) \times L} \\
\mathbf{0}_{L \times L} & \mathbf{0}_{L \times L} & \mathbf{0}_{\left(N_{d}-L\right) \times\left(N_{d}-L\right)} & \boldsymbol{I}_{L}
\end{array}\right] .
\end{aligned}
$$

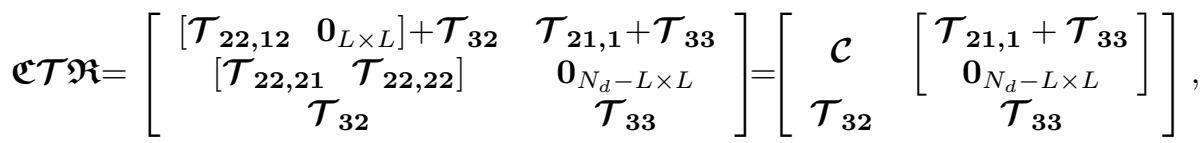

Here, having the matrix $\mathcal{T}_{\mathbf{3 2 , 2}}$ on top right corner of $\mathcal{H}$ makes it circulant. In addition, the matrix $\mathcal{C}_{\text {ISI }}=\mathcal{T}_{\mathbf{2 1}, \mathbf{1}}+\mathcal{T}_{\mathbf{3 3}}$ which also emulates a circulant channel on the first $L$ samples of $\vec{x}_{u}$-causes ISI into the first $L$ samples of $\vec{y}_{d}$. However, since both $\vec{x}_{u}$ and $\hat{\vec{h}}$ are known, one can simply remove the known interference, i.e. (27). We also note that, since the matrix $\mathfrak{C}$ is located at the receiver side, the first $L$ samples of $\vec{w}_{u}$ would also be added to the first $L$ samples of $\vec{w}_{d}$. If $N_{d}>>L$, the noise correlation would be negligible. Nonetheless, the operation (26) provides the significant advantage of designing payload and UW sequences separately, and thus performing $N_{d}$-point FFT only on the received payload signal.

\section{REFERENCES}

[1] 3GPP, "NR; NR and NG-RAN Overall Description; Stage 2, Rel. 15," 3rd Generation Partnership Project (3GPP); Tech. Spec. Group Radio Access Network, Technical Specification (TS) 38.300, 06 2018, v15.2.0.

[2] L. Deneire, B. Gyselinckx, and M. Engels, "Training sequence vs. cyclic prefix a new look on single carrier communication," in Globecom'00IEEE. Global Telecommunications Conference. Conference Record (Cat. No. OOCH37137), vol. 2. IEEE, 2000, pp. 1056-1060.

[3] H. Witschnig, T. Mayer, A. Springer, A. Koppler, L. Maurer, M. Huemer, and R. Weigel, "A different look on cyclic prefix for SC/FDE," in The 13th IEEE International Symposium on Personal, Indoor and Mobile Radio Communications, vol. 2. IEEE, 2002, pp. 824-828.

[4] J. Coon, M. Sandell, M. Beach, and J. McGeehan, "Channel and Noise Variance Estimation and Tracking Algorithms for Unique-Word based Single-Carrier Systems," IEEE Trans. on Wireless Commun., vol. 5, no. 6, pp. 1488-1496, 2006.

[5] M. Huemer, H. Witschnig, and J. Hausner, "Unique Word Based Phase Tracking Algorithms for SC/FDE-Systems," in GLOBECOM'03. IEEE Global Telecommunications Conference, vol. 1. IEEE, 2003, pp. 70-74.

[6] A. Sahin, R. Yang, M. Ghosh, and R. L. Olesen, "An Improved Unique Word DFT-spread OFDM Scheme for 5G Systems," in 2015 IEEE Globecom Workshops (GC Wkshps). IEEE, 2015, pp. 1-6.

[7] A. Sahin, R. Yang, E. Bala, M. C. Beluri, and R. L. Olesen, "Flexible DFT-S-OFDM: solutions and challenges," IEEE Communications Magazine, vol. 54, no. 11, pp. 106-112, 2016.

[8] M. Huemer, C. Hofbauer, A. Onic, and J. B. Huber, "Design and analysis of UW-OFDM signals," AEU - International Journal of Electronics and Communications, vol. 68, no. 10, pp. 958 - 968, 2014

[9] J. T. Dias and R. C. de Lamare, "Unique-Word GFDM Transmission Systems," IEEE Wireless Commun. Lett., vol. 6, no. 6, pp. 746-749, 2017.

[10] D. Zhang, A. Festag, and G. P. Fettweis, "Performance of Generalized Frequency Division Multiplexing Based Physical Layer in Vehicular Communications," IEEE Trans. on Vehicular Technology, vol. 66, no. 11, pp. 9809-9824, 2017.

[11] S. Ehsanfar, M. Matthé, D. Zhang, and G. Fettweis, "A Study of PilotAided Channel Estimation in MIMO-GFDM Systems," in Proc. of the ITG/IEEE Workshop on Smart Antennas (WSA'16), 2016.

[12] S. A. Cheema, J. Zhang, M. Huemer, and M. Haardt, "Linear detection schemes for MIMO UW-OFDM," in 2016 50th Asilomar Conference on Signals, Systems and Computers. IEEE, 2016, pp. 1457-1461.
[13] V. Tomashevich and I. Polian, "Detection Performance of MIMO Unique Word OFDM," in WSA 2016; 20th International ITG Workshop on Smart Antennas. VDE, 2016, pp. 1-8.

[14] S. Ehsanfar, M. Chafii, and G. Fettweis, "A Frame Design for MIMO UW based Systems: Overhead Analysis \& Channel Estimation," in 2019 IEEE 2nd 5G World Forum (5GWF), Sep. 2019, pp. 173-178.

[15] A. H. Roger and R. J. Charles, "Topics in matrix analysis," 1991.

[16] X. Liu, H.-H. Chen, S. Chen, and W. Meng, "Symbol Cyclic-Shift Equalization Algorithm-A CP-Free OFDM/OFDMA System Design," IEEE Trans. Veh. Technol., vol. 66, no. 1, pp. 282-294, 2016.

[17] T.-D. Chiueh and P.-Y. Tsai, OFDM baseband receiver design for wireless communications. John Wiley \& Sons, 2008

[18] A. N. Mody and G. L. Stuber, "Synchronization for MIMO OFDM systems," in IEEE Global Telecommunications Conference (GLOBECOM'01), vol. 1. IEEE, 2001, pp. 509-513.

[19] N. Suehiro and M. Hatori, "Modulatable orthogonal sequences and their application to SSMA systems," IEEE Trans. Inf. Theory, vol. 34, no. 1 pp. 93-100, 1988

[20] N. Michailow et. al., "Generalized Frequency Division Multiplexing for 5th Generation Cellular Networks," IEEE Trans. Commun., vol. 62 , no. 9, pp. 3045-3061, 2014.

[21] S. M. Kay, Fundamentals of Statistical Signal Processing: Estimation Theory. Upper Saddle River, NJ: Prentice Hall, 1993, vol. 1.

[22] S. Ehsanfar, M. Matthé, M. Chafii, and G. Fettweis, "Pilot- and CPAided Channel Estimation in MIMO Non-Orthogonal Multi-Carriers," IEEE Trans. on Wireless Commun., vol. 18, no. 1, pp. 650-664, Jan 2019.

[23] S. Ehsanfar, M. Chafii, and G. Fettweis, "Time-Variant Pilot- and CPAided Channel Estimation for GFDM," in Proc. of the IEEE International Conference on Commun. (ICC'19). IEEE, 2019.

[24] M. Huemer, A. Onic, and C. Hofbauer, "Classical and Bayesian linear data estimators for unique word OFDM," IEEE Trans. on Signal Processing, vol. 59, no. 12, pp. 6073-6085, 2011.

[25] M. Huemer and O. Lang, "On component-wise conditionally unbiased linear Bayesian estimation," in Proc. of 48th Asilomar Conference on Signals, Systems and Computers, 2014.

[26] A. Nimr, M. Matthé, D. Zhang, and G. Fettweis, "Optimal Radix-2 FFT Compatible Filters for GFDM," IEEE Commun. Lett., vol. 21, no. 7, pp. 1497-1500, 2017.

[27] R. H. Clarke, "A statistical theory of mobile-radio reception," The Bell System Technical Journal, vol. 47, no. 6, pp. 957-1000, July 1968.

[28] M. Matthé, D. Zhang, and G. Fettweis, "Low-Complexity Iterative MMSE-PIC Detection for MIMO-GFDM," IEEE Trans. on Commun., vol. 66, no. 4, pp. 1467-1480, April 2018.

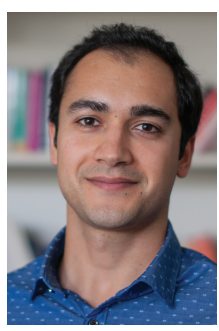

Shahab Ehsanfar received the B.Sc. and M.Sc degrees in electronics engineering from Azad university, Iran, in 2010, and the Politecnico di Torino, Italy, in 2014, respectively. In 2013, he received a scholarship for the master's thesis from Politecnico di Torino, where he conducted research with RWTH Aachen, Germany. In the master's thesis, he focused on multi-user resource allocation under semipersistent scheduling in interference-limited LTE networks. He joined the Vodafone Chair Mobile Communications Systems, Technische Universität Dresden, in 2015 and was granted a Ph.D. degree in January 2020. His research interests include pilot design, MIMO channel estimation, and data detection for non-orthogonal multi-carrier systems. 


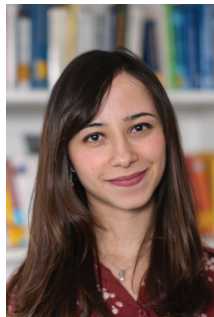

Marwa Chafii received the $\mathrm{Ph} . \mathrm{D}$. degree in telecommunications from Centrale Supélec France, in 2016. After that, she joined the TU Dresden Vodafone Chair Mobile Communications Systems, Germany, as a Research Group Leader. Since September 2018 she is research projects lead at Women in AI and an associate professor at ENSEA, France, where she holds a Chair of Excellence from Paris Seine Initiative, in 2018. Her research interests include signal processing for digital communications, advanced waveform design for wireless communications, and machine learning for communications. She currently serves as Associate Editor at IEEE Communications Letters, and she is vice-chair of the IEEE ComSoc Emerging Technology Initiative on Machine Learning for Communications. She is also currently managing the Gender Committee of the AI4EU community.

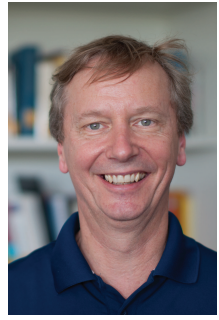

Gerhard P. Fettweis is Vodafone Chair Professor at TU Dresden since 1994, and heads the Barkhausen Institute since 2018, respectively. He earned his Ph.D. under H. Meyr's supervision from RWTH Aachen in 1990. After one year at IBM Research in San Jose, CA, he moved to TCSI Inc., Berkeley, CA. He coordinates the $5 \mathrm{G}$ Lab Germany, and 2 German Science Foundation (DFG) centers at TU Dresden, namely cfaed and HAEC. His research focusses on wireless transmission and chip design for wireless/IoT platforms, with 20 companies from Asia/Europe/US sponsoring his research. Gerhard is IEEE Fellow, membe of the German Academy of Sciences (Leopoldina), the German Academy of Engineering (acatech), and received multiple IEEE recognitions as well has the VDE ring of honor. In Dresden his team has spun-out sixteen start-ups, and setup funded projects in volume of close to EUR 1/2 billion. He co-chairs the IEEE 5G Initiative, and has helped organizing IEEE conferences, most notably as TPC Chair of ICC 2009 and of TTM 2012, and as General Chair of VTC Spring 2013 and DATE 2014. 